DESY 05-038

October 19, 2018

\title{
Exclusive electroproduction of $\phi$ mesons at HERA
}

\author{
ZEUS Collaboration
}

\begin{abstract}
Exclusive electroproduction of $\phi$ mesons has been studied in $e^{ \pm} p$ collisions at $\sqrt{s}=318 \mathrm{GeV}$ with the ZEUS detector at HERA using an integrated luminosity of $65.1 \mathrm{pb}^{-1}$. The $\gamma^{*} p$ cross section is presented in the kinematic range $2<Q^{2}<$ $70 \mathrm{GeV}^{2}, 35<W<145 \mathrm{GeV}$ and $|t|<0.6 \mathrm{GeV}^{2}$. The cross sections as functions of $Q^{2}, W, t$ and helicity angle $\theta_{h}$ are compared to cross sections for other vector mesons. The ratios $R$ of the cross sections for longitudinally and transversely polarized virtual photons are presented as functions of $Q^{2}$ and $W$. The data are also compared to predictions from theoretical models.
\end{abstract}




\section{The ZEUS Collaboration}

S. Chekanov, M. Derrick, S. Magill, S. Miglioranzi ${ }^{1}$, B. Musgrave, J. Repond, R. Yoshida Argonne National Laboratory, Argonne, Illinois 60439-4815, USA ${ }^{n}$

M.C.K. Mattingly

Andrews University, Berrien Springs, Michigan 49104-0380, USA

N. Pavel, A.G. Yagües Molina

Institut für Physik der Humboldt-Universität zu Berlin, Berlin, Germany

P. Antonioli, G. Bari, M. Basile, L. Bellagamba, D. Boscherini, A. Bruni, G. Bruni,

G. Cara Romeo, L. Cifarelli, F. Cindolo, A. Contin, M. Corradi, S. De Pasquale, P. Giusti,

G. Iacobucci, A. Margotti, A. Montanari, R. Nania, F. Palmonari, A. Pesci, A. Polini,

L. Rinaldi, G. Sartorelli, A. Zichichi

University and INFN Bologna, Bologna, Italy ${ }^{e}$

G. Aghuzumtsyan, D. Bartsch, I. Brock, S. Goers, H. Hartmann, E. Hilger, P. Irrgang, H.-P. Jakob, O.M. Kind, U. Meyer, E. Paul' ${ }^{2}$, J. Rautenberg, R. Renner, K.C. Voss ${ }^{3}$, M. Wang, M. Wlasenko

Physikalisches Institut der Universität Bonn, Bonn, Germany ${ }^{b}$

D.S. Bailey ${ }^{4}$, N.H. Brook, J.E. Cole, G.P. Heath, T. Namsoo, S. Robins

H.H. Wills Physics Laboratory, University of Bristol, Bristol, United Kingdom ${ }^{m}$

M. Capua, S. Fazio, A. Mastroberardino, M. Schioppa, G. Susinno, E. Tassi

Calabria University, Physics Department and INFN, Cosenza, Italy ${ }^{e}$

J.Y. Kim, K.J. Ma ${ }^{5}$

Chonnam National University, Kwangju, South Korea ${ }^{g}$

M. Helbich, Y. Ning, Z. Ren, W.B. Schmidke, F. Sciulli

Nevis Laboratories, Columbia University, Irvington on Hudson, New York 10027o

J. Chwastowski, A. Eskreys, J. Figiel, A. Galas, K. Olkiewicz, P. Stopa, D. Szuba, L. Zawiejski

Institute of Nuclear Physics, Cracow, Poland ${ }^{i}$

L. Adamczyk, T. Bołd, I. Grabowska-Bołd, D. Kisielewska, A.M. Kowal, J. Łukasik, M. Przybycień, L. Suszycki, J. Szuba ${ }^{6}$

Faculty of Physics and Applied Computer Science, AGH-University of Science and Technology, Cracow, Poland ${ }^{p}$

A. Kotański ${ }^{7}$, W. Słomiński

Department of Physics, Jagellonian University, Cracow, Poland 
V. Adler, U. Behrens, I. Bloch, K. Borras, G. Drews, J. Fourletova, A. Geiser, D. Gladkov, P. Göttlicher ${ }^{8}$, O. Gutsche, T. Haas, W. Hain, C. Horn, B. Kahle, U. Kötz, H. Kowalski, G. Kramberger, D. Lelas ${ }^{9}$, H. Lim, B. Löhr, R. Mankel, I.-A. Melzer-Pellmann, C.N. Nguyen, D. Notz, A.E. Nuncio-Quiroz, A. Raval, R. Santamarta, U. Schneekloth, H. Stadie, U. Stösslein, G. Wolf, C. Youngman, W. Zeuner

Deutsches Elektronen-Synchrotron DESY, Hamburg, Germany

S. Schlenstedt

Deutsches Elektronen-Synchrotron DESY, Zeuthen, Germany

G. Barbagli, E. Gallo, C. Genta, P. G. Pelfer

University and INFN, Florence, Italy ${ }^{e}$

A. Bamberger, A. Benen, F. Karstens, D. Dobur, N.N. Vlasov ${ }^{10}$

Fakultät für Physik der Universität Freiburg i.Br., Freiburg i.Br., Germany ${ }^{b}$

P.J. Bussey, A.T. Doyle, W. Dunne, J. Ferrando, J. Hamilton, D.H. Saxon, I.O. Skillicorn

Department of Physics and Astronomy, University of Glasgow, Glasgow, United King$\mathrm{dom}^{\mathrm{m}}$

I. Gialas ${ }^{11}$

Department of Engineering in Management and Finance, Univ. of Aegean, Greece

T. Carli ${ }^{12}$, T. Gosau, U. Holm, N. Krumnack ${ }^{13}$, E. Lohrmann, M. Milite, H. Salehi, P. Schleper, T. Schörner-Sadenius, S. Stonjek ${ }^{14}$, K. Wichmann, K. Wick, A. Ziegler, Ar. Ziegler

Hamburg University, Institute of Exp. Physics, Hamburg, Germany ${ }^{b}$

C. Collins-Tooth ${ }^{15}$, C. Foudas, C. Fry, R. Gonçalo ${ }^{16}$, K.R. Long, A.D. Tapper

Imperial College London, High Energy Nuclear Physics Group, London, United Kingdom $^{m}$

M. Kataoka ${ }^{17}$, K. Nagano, K. Tokushuku ${ }^{18}$, S. Yamada, Y. Yamazaki

Institute of Particle and Nuclear Studies, KEK, Tsukuba, Japan ${ }^{f}$

A.N. Barakbaev, E.G. Boos, N.S. Pokrovskiy, B.O. Zhautykov

Institute of Physics and Technology of Ministry of Education and Science of Kazakhstan, Almaty, Kazakhstan

D. Son

Kyungpook National University, Center for High Energy Physics, Daegu, South Korea ${ }^{g}$

J. de Favereau, K. Piotrzkowski

Institut de Physique Nucléaire, Université Catholique de Louvain, Louvain-la-Neuve, Belgium $^{q}$ 
F. Barreiro, C. Glasman ${ }^{19}$, M. Jimenez, L. Labarga, J. del Peso, J. Terrón, M. Zambrana Departamento de Física Teórica, Universidad Autónoma de Madrid, Madrid, Spain ${ }^{l}$

F. Corriveau, C. Liu, M. Plamondon, A. Robichaud-Veronneau, R. Walsh, C. Zhou Department of Physics, McGill University, Montréal, Québec, Canada H3A 2T8 ${ }^{a}$

T. Tsurugai

Meiji Gakuin University, Faculty of General Education, Yokohama, Japan ${ }^{f}$

A. Antonov, B.A. Dolgoshein, I. Rubinsky, V. Sosnovtsev, A. Stifutkin, S. Suchkov Moscow Engineering Physics Institute, Moscow, Russia ${ }^{j}$

R.K. Dementiev, P.F. Ermolov, L.K. Gladilin, I.I. Katkov, L.A. Khein, I.A. Korzhavina, V.A. Kuzmin, B.B. Levchenko, O.Yu. Lukina, A.S. Proskuryakov, L.M. Shcheglova, D.S. Zotkin, S.A. Zotkin

Moscow State University, Institute of Nuclear Physics, Moscow, Russia ${ }^{k}$

I. Abt, C. Büttner, A. Caldwell, X. Liu, J. Sutiak

Max-Planck-Institut für Physik, München, Germany

N. Coppola, G. Grigorescu, A. Keramidas, E. Koffeman, P. Kooijman, E. Maddox, H. Tiecke, M. Vázquez, L. Wiggers

NIKHEF and University of Amsterdam, Amsterdam, Netherlands ${ }^{h}$

N. Brümmer, B. Bylsma, L.S. Durkin, T.Y. Ling

Physics Department, Ohio State University, Columbus, Ohio $43210^{n}$

P.D. Allfrey, M.A. Bell, A.M. Cooper-Sarkar, A. Cottrell, R.C.E. Devenish, B. Foster, G. Grzelak, C. Gwenlan ${ }^{20}$, T. Kohno, S. Patel, P.B. Straub, R. Walczak

Department of Physics, University of Oxford, Oxford United Kingdom ${ }^{m}$

P. Bellan, A. Bertolin, R. Brugnera, R. Carlin, R. Ciesielski, F. Dal Corso, S. Dusini, A. Garfagnini, S. Limentani, A. Longhin, L. Stanco, M. Turcato

Dipartimento di Fisica dell' Università and INFN, Padova, Italy ${ }^{e}$

E.A. Heaphy, F. Metlica, B.Y. Oh, J.J. Whitmore ${ }^{21}$

Department of Physics, Pennsylvania State University, University Park, Pennsylvania $16802^{\circ}$

Y. Iga

Polytechnic University, Sagamihara, Japan ${ }^{f}$

G. D'Agostini, G. Marini, A. Nigro

Dipartimento di Fisica, Università 'La Sapienza' and INFN, Rome, Italy ${ }^{e}$

J.C. Hart

Rutherford Appleton Laboratory, Chilton, Didcot, Oxon, United Kingdom ${ }^{m}$ 
H. Abramowicz ${ }^{22}$, A. Gabareen, S. Kananov, A. Kreisel, A. Levy

Raymond and Beverly Sackler Faculty of Exact Sciences, School of Physics, Tel-Aviv

University, Tel-Aviv, Israel ${ }^{d}$

M. Kuze

Department of Physics, Tokyo Institute of Technology, Tokyo, Japan ${ }^{f}$

S. Kagawa, T. Tawara

Department of Physics, University of Tokyo, Tokyo, Japan ${ }^{f}$

R. Hamatsu, H. Kaji, S. Kitamura ${ }^{23}$, K. Matsuzawa, O. Ota, Y.D. Ri

Tokyo Metropolitan University, Department of Physics, Tokyo, Japan ${ }^{f}$

M. Costa, M.I. Ferrero, V. Monaco, R. Sacchi, A. Solano

Università di Torino and INFN, Torino, Italy $^{e}$

M. Arneodo, M. Ruspa

Università del Piemonte Orientale, Novara, and INFN, Torino, Italy ${ }^{e}$

S. Fourletov, J.F. Martin

Department of Physics, University of Toronto, Toronto, Ontario, Canada M5S 1A7\%a

J.M. Butterworth ${ }^{24}$, R. Hall-Wilton, T.W. Jones, J.H. Loizides ${ }^{25}$, M.R. Sutton ${ }^{4}$, C. TargettAdams, M. Wing

Physics and Astronomy Department, University College London, London, United Kingdom $^{m}$

J. Ciborowski ${ }^{26}$, P. Kulinski, P. Łużniak ${ }^{27}$, J. Malka ${ }^{27}$, R.J. Nowak, J.M. Pawlak, J. Sztuk ${ }^{28}$, T. Tymieniecka, A. Tyszkiewicz ${ }^{27}$, A. Ukleja, J. Ukleja ${ }^{29}$, A.F. Żarnecki

Warsaw University, Institute of Experimental Physics, Warsaw, Poland

M. Adamus, P. Plucinski

Institute for Nuclear Studies, Warsaw, Poland

Y. Eisenberg, D. Hochman, U. Karshon, M.S. Lightwood

Department of Particle Physics, Weizmann Institute, Rehovot, Israel ${ }^{c}$

E. Brownson, T. Danielson, A. Everett, D. Kçira, S. Lammers, L. Li, D.D. Reeder, M. Rosin, P. Ryan, A.A. Savin, W.H. Smith

Department of Physics, University of Wisconsin, Madison, Wisconsin 53706, USA ${ }^{n}$

S. Dhawan

Department of Physics, Yale University, New Haven, Connecticut 06520-8121, USA ${ }^{n}$

S. Bhadra, C.D. Catterall, Y. Cui, G. Hartner, S. Menary, U. Noor, M. Soares, J. Standage, J. Whyte

Department of Physics, York University, Ontario, Canada M3J 1P3 ${ }^{a}$ 
1 also affiliated with University College London, UK

2 retired

${ }^{3}$ now at the University of Victoria, British Columbia, Canada

4 PPARC Advanced fellow

${ }^{5}$ supported by a scholarship of the World Laboratory Björn Wiik Research Project

${ }^{6}$ partly supported by Polish Ministry of Scientific Research and Information Technology, grant no.2P03B 12625

7 supported by the Polish State Committee for Scientific Research, grant no. 2 P03B 09322

8 now at DESY group FEB, Hamburg, Germany

9 now at LAL, Université de Paris-Sud, IN2P3-CNRS, Orsay, France

10 partly supported by Moscow State University, Russia

11 also affiliated with DESY

12 now at CERN, Geneva, Switzerland

13 now at Baylor University, USA

${ }^{14}$ now at University of Oxford, UK

15 now at the Department of Physics and Astronomy, University of Glasgow, UK

16 now at Royal Holloway University of London, UK

17 also at Nara Women's University, Nara, Japan

18 also at University of Tokyo, Japan

19 Ramón y Cajal Fellow

${ }^{20}$ PPARC Postdoctoral Research Fellow

${ }^{21}$ on leave of absence at The National Science Foundation, Arlington, VA, USA

22 also at Max Planck Institute, Munich, Germany, Alexander von Humboldt Research Award

23 present address: Tokyo Metropolitan University of Health Sciences, Tokyo 116-8551, Japan

24 also at University of Hamburg, Germany, Alexander von Humboldt Fellow

${ }^{25}$ partially funded by DESY

26 also at Eódź University, Poland

27 Łódź University, Poland

${ }^{28}$ Eódź University, Poland, supported by the KBN grant 2P03B12925

${ }^{29}$ supported by the KBN grant 2P03B12725 
a supported by the Natural Sciences and Engineering Research Council of Canada (NSERC)

$b$ supported by the German Federal Ministry for Education and Research (BMBF), under contract numbers HZ1GUA 2, HZ1GUB 0, HZ1PDA 5, HZ1VFA 5

c supported in part by the MINERVA Gesellschaft für Forschung GmbH, the Israel Science Foundation (grant no. 293/02-11.2), the U.S.-Israel Binational Science Foundation and the Benozyio Center for High Energy Physics

$d$ supported by the German-Israeli Foundation and the Israel Science Foundation

$e$ supported by the Italian National Institute for Nuclear Physics (INFN)

$f$ supported by the Japanese Ministry of Education, Culture, Sports, Science and Technology (MEXT) and its grants for Scientific Research

$g$ supported by the Korean Ministry of Education and Korea Science and Engineering Foundation

$h$ supported by the Netherlands Foundation for Research on Matter (FOM)

$i$ supported by the Polish State Committee for Scientific Research, grant no. 620/E-77/SPB/DESY/P-03/DZ 117/2003-2005 and grant no. 1P03B07427/2004-2006

$j$ partially supported by the German Federal Ministry for Education and Research (BMBF)

$k$ supported by RF Presidential grant N 1685.2003.2 for the leading scientific schools and by the Russian Ministry of Education and Science through its grant for Scientific Research on High Energy Physics

$l$ supported by the Spanish Ministry of Education and Science through funds provided by CICYT

$m$ supported by the Particle Physics and Astronomy Research Council, UK

$n$ supported by the US Department of Energy

$o$ supported by the US National Science Foundation

$p$ supported by the Polish Ministry of Scientific Research and Information Technology, grant no. 112/E-356/SPUB/DESY/P-03/DZ 116/2003-2005 and 1 P03B 06527

$q$ supported by FNRS and its associated funds (IISN and FRIA) and by an Inter-University Attraction Poles Programme subsidised by the Belgian Federal Science Policy Office 


\section{Introduction}

Exclusive electroproduction of vector mesons is a process which can be used to confront model predictions in a kinematic region that includes the transition between soft and hard dynamics. The process also lends itself to detailed experimental investigation; the decay products of the vector meson can be precisely measured so that distributions in several variables can be studied with high resolution over a large phase space.

Many measurements of exclusive production of vector mesons, e $p \rightarrow e V p$, have been made at HERA [1-16]. In this paper, results from a study of exclusive $\phi$ production are presented. The data sample represents a factor $\sim 30$ increase over previously published HERA results $[9,10]$. The measurements were made for virtuality of the exchanged photon, $Q^{2}$, in the range $2<Q^{2}<70 \mathrm{GeV}^{2}$ and for the photon-proton center-of-mass energy, $W$, in the range $35<W<145 \mathrm{GeV}$. The cross sections are presented as functions of $Q^{2}, W$, the squared four-momentum transfer at the proton vertex, $t$, and helicity angle $\theta_{h}$. The $W$ dependence was also extracted in bins of $t$, and the ratio, $R$, of the cross sections for longitudinally and transversely polarized virtual photons, determined from the angular distribution of the decay products of the $\phi$ mesons, is presented as functions of $W$ and $Q^{2}$.

\section{Phenomenology}

A simple picture for the process $e p \rightarrow e V p$, where $V$ represents a vector meson, can be formulated in the rest frame of the proton. In this frame, the virtual photon emitted from the electron fluctuates into a quark-antiquark dipole, which then scatters elastically off the proton. Long after the interaction, the $q \bar{q}$ pair forms a vector meson. It can be shown that, at high energy, the $q \bar{q}$ creation, scattering, and vector meson formation are well separated in time so that the cross section for the process can be factorized into terms representing the $q \bar{q}$ coupling to the photon, the dipole scattering cross section on the proton, and the final-state formation $[17,18]$. The dipole scattering cross section on the proton depends on the transverse separation of the $q \bar{q}$ pair in the dipole. The interactions of large dipoles are thought to be primarily 'soft' and therefore described by Regge phenomenology [19]. On the other hand, the interactions of dipoles with small transverse separation of the $q \bar{q}$ pair are expected to be 'hard', and therefore calculable in perturbative QCD (pQCD). In lowest-order pQCD, the process proceeds via the exchange of two gluons between the proton and the $q \bar{q}$ dipole so that vector-meson production is sensitive to the gluon density in the proton [20].

The transverse size of the dipole is related to the transverse-energy scale of the interaction, 
and can depend on $Q$ as well as on the quark mass, $M$ [21]. Large transverse-energy scales preferentially select small dipole sizes. The impact parameter for the dipole scattering on the proton depends on $\sqrt{|t|}$. Large $|t|$ preferentially selects small impact parameters. The $|t|$ values in this analysis are small and no pQCD predictions are possible for the $|t|$ dependence in this regime. The pQCD predictions for the $W$ and $Q^{2}$ dependence of the cross section should be more accurate as either or both of $Q$ or $M$ become large.

The pQCD predictions depend on the square of the gluon density, leading to a steep dependence of the $\gamma^{*} p$ cross section on $W$, in contrast to expectations from Regge phenomenology. For example, a gluon density varying as $x g(x) \propto x^{-0.2}$, where $x$ is the Bjorken scaling variable, would lead to $\sigma\left(\gamma^{*} p \rightarrow V p\right) \propto W^{0.8}$. The Regge phenomenology expectation is $\sigma\left(\gamma^{*} p \rightarrow V p\right) \propto W^{0.2}$. The energy dependence of the $\gamma^{*} p$ cross section is therefore a good indicator of whether gluon exchange is dominant. Data from exclusive $\rho$ production $[2,4,5]$ show that the cross section $\sigma\left(\gamma^{*} p \rightarrow \rho p\right)$ rises with $W$ as $W^{\delta}$, where $\delta$ increases with $Q^{2}$ from about 0.2 at $Q^{2}=0$ (photoproduction) to about 0.7 at $Q^{2} \approx 30 \mathrm{GeV}^{2}$. However, in the case of exclusive $J / \psi$ production the cross section rises steeply with $W$ even for photoproduction $[12,13,16]$. The investigation of elastic $\phi$ production is of particular interest since the $\phi$ is an $s \bar{s}$ state, and the mass of the strange quark is not negligible in pQCD calculations. Experimentally, the extraction of the $\phi$ signal is very clean due to the narrow width of the state.

The measurement of the variation of the $W$ dependence of the cross section with $t$ also provides a good test of the validity of pQCD calculations. In pQCD, little variation is expected, while for soft hadronic processes the $W$ dependence varies as $\sigma \propto W^{4\left(\alpha_{\mathbb{P}}(t)-1\right)}$, with $\alpha_{\mathbb{P}}(t)=1.08+0.25 t[22,23]$.

Specific predictions for $\phi$ meson production are available in the dipole model realization (FS04) of Forshaw and Shaw [24]. The predictions depend on the strange-quark mass used in the photon wavefunction, on the parametrization of the dipole-proton scattering cross section, and on the vector-meson wavefunction. The gluon density does not appear explicitly but is implicit in the dipole scattering cross section. The predictions shown in this paper employed a strange-quark mass of $200 \mathrm{MeV}$, and a Gaussian wave-function for the $\phi$-meson [25]. The dipole-proton cross section is obtained from the best fit to the total $\gamma^{*} p$ cross section [24] and implies a saturation of the cross section as $x$ decreases.

Specific predictions for the $\phi$ meson are also available in the model of Martin, Ryskin and Teubner (MRT) [26]. In the MRT model, the properties of the event are determined completely from the features of the photon wavefunction and the gluon density in the proton. Parton-hadron duality is invoked to relate the parton-level cross section to the cross section for the produced vector meson.

As NLO corrections are not fully taken into account, the model calculations come with 
significant normalization uncertainties.

The data of this analysis are also compared to results from other vector mesons. The results are presented as a function of $Q^{2}+M_{V}^{2}$, where $M_{V}$ is the vector meson mass, for cross sections, and as a function of $Q^{2} / M_{V}^{2}$ for the spin-density matrix element, $r_{00}^{04}$, to test for scaling in these variables.

A recent review of models and data can be consulted for more detailed information on vector meson production [27].

\section{$3 \quad$ Experimental set-up}

The data were collected during 1998-2000 with the ZEUS detector and correspond to an integrated luminosity of $15.0 \mathrm{pb}^{-1}$ for $e^{-} p$ and $50.1 \mathrm{pb}^{-1}$ for $e^{+} p$ collisions with a proton energy of $920 \mathrm{GeV}$ and an $e^{ \pm}$energy of $27.5 \mathrm{GeV}$. Since no dependence on the lepton charge is expected, the two data sets were combined ${ }^{1}$. A detailed description of the ZEUS detector can be found elsewhere [28]. A brief outline of the components that are most relevant for this analysis is given below.

Charged particles were reconstructed in the central tracking detector (CTD) [29] covering the polar-angle ${ }^{2}$ region $15^{\circ}<\theta<164^{\circ}$. The transverse-momentum resolution for fulllength tracks is $\sigma\left(p_{T}\right) / p_{T}=0.0058 p_{T} \oplus 0.0065 \oplus 0.0014 / p_{T}$, with $p_{T}$ in $\mathrm{GeV}$.

The high-resolution uranium calorimeter (CAL) [30] consists of three parts: the forward (FCAL), the barrel (BCAL) and the rear (RCAL) calorimeters. Each part is subdivided transversely into towers and longitudinally into an electromagnetic section (EMC) and either one (RCAL) or two (FCAL and BCAL) hadronic sections. The CAL covers $99.7 \%$ of the total solid angle. Under test-beam conditions, the CAL single-particle relative energy resolution is $\sigma(E) / E=0.18 / \sqrt{E}$ for electrons and $\sigma(E) / E=0.35 / \sqrt{E}$ for hadrons, with $E$ in $\mathrm{GeV}$.

The forward plug calorimeter (FPC) [31] was a lead-scintillator sandwich calorimeter with readout via wavelength-shifter fibers. It was installed in the beamhole of the FCAL and extended the pseudorapidity coverage of the forward calorimeter from $\eta \lesssim 4$ to $\eta \lesssim 5$. It has since been removed to accomodate HERA magnets for the high-luminosity HERA II run.

\footnotetext{
${ }^{1}$ Hereafter, both $e^{+}$and $e^{-}$are referred to as electrons, unless explicitly stated otherwise.

${ }^{2}$ The ZEUS coordinate system is a right-handed Cartesian system, with the $Z$ axis pointing in the proton beam direction, referred to as the "forward direction", and the $X$ axis pointing left towards the center of HERA. The coordinate origin is at the nominal interaction point.
} 
The small-angle rear tracking detector (SRTD) [32] consists of two planes of scintillator strips read out via optical fibers. It is attached to the front face of the RCAL and covers an angular range between $162^{\circ}<\theta<176^{\circ}$. The SRTD provides a transverse position resolution for the scattered electron of $0.3 \mathrm{~cm}$ [12] corresponding to an angular resolution of $2 \mathrm{mrad}$.

The hadron-electron separator installed in the RCAL (RHES) consists of silicon diodes placed at a longitudinal depth of three radiation lengths. The RHES provides an electron position resolution of $0.5 \mathrm{~cm}$ if at least two adjacent pads are hit by the shower [33].

The luminosity was determined from the rate of the bremsstrahlung process $e p \rightarrow e \gamma p$, where the photon was measured with a lead-scintillator calorimeter [34] placed in the HERA tunnel at $Z=-107 \mathrm{~m}$ in the HERA tunnel.

\section{$4 \quad$ Kinematics and cross sections}

The following kinematic variables are used to describe exclusive $\phi$ production,

$$
e(k) p(P) \rightarrow e\left(k^{\prime}\right) \phi(v) p\left(P^{\prime}\right),
$$

where $k, k^{\prime}, P, P^{\prime}$ and $v$ are, respectively, the four-momenta of the incident electron, scattered electron, incident proton, scattered proton and the $\phi$ meson:

- $Q^{2}=-q^{2}=-\left(k-k^{\prime}\right)^{2}$, the negative four-momentum squared of the virtual photon;

- $W^{2}=(q+P)^{2}$, the squared invariant mass of the photon-proton system;

- $y=(P \cdot q) /(P \cdot k)$, the fraction of the electron energy transferred to the proton in the proton rest frame;

- $x=Q^{2} /(2 P \cdot q)$, the Bjorken variable;

- $t=\left(P-P^{\prime}\right)^{2}$, the squared four-momentum transfer at the proton vertex.

The kinematic variables were reconstructed with the "constrained" method [2] which uses the momentum vector of the $\phi$ and the polar and azimuthal angles of the scattered electron.

The $e p$ cross section can be expressed in terms of the transverse, $\sigma_{T}$, and longitudinal, $\sigma_{L}$, virtual photoproduction cross sections as

$$
\frac{d^{2} \sigma^{e p \rightarrow e \phi p}}{d y d Q^{2}}=\Gamma_{T}\left(y, Q^{2}\right)\left(\sigma_{T}+\epsilon \sigma_{L}\right)
$$

where $\Gamma_{T}$ is the flux of transverse virtual photons [35] and $\epsilon$ is the ratio of longitudinal and transverse virtual-photon fluxes, given by $\epsilon=2(1-y) /\left(1+(1-y)^{2}\right)$. In the kinematic range studied here, $\epsilon$ lies in the range $0.975<\epsilon<1$, with an average value of 0.99 . 
The virtual photon-proton cross section, $\sigma^{\gamma^{*} p \rightarrow \phi p} \equiv \sigma_{T}+\epsilon \sigma_{L}$, can be used to evaluate the total exclusive cross section, $\sigma_{\text {tot }}^{\gamma^{*} p \rightarrow \phi p} \equiv \sigma_{T}+\sigma_{L}$, through the relation

$$
\sigma_{\mathrm{tot}}^{\gamma^{*} p \rightarrow \phi p}=\frac{1+R}{1+\epsilon R} \sigma^{\gamma^{*} p \rightarrow \phi p}
$$

where $R=\sigma_{L} / \sigma_{T}$ is the ratio of the cross sections for longitudinal and transverse photons. The helicity structure of $\phi$ production is used to determine $R$ as described in Section 8.5.1.

\section{$5 \quad$ Reconstruction and selection of events}

The signature of exclusive $\phi$ electroproduction, $e p \rightarrow e \phi p$, consists of the scattered electron and two oppositely charged kaons from the $\phi$ decay. The scattered proton is deflected through a small angle and escapes undetected down the beampipe. The data selection and analysis are described in detail elsewhere [36]. A brief outline is given here. The events were selected online by a three-level trigger $[37,38]$. The trigger required a scattered electron in the CAL with energy greater than $7 \mathrm{GeV}$, a minimum of two and a maximum of five tracks reconstructed with the CTD and less than $5 \mathrm{GeV}$ in the towers of the FCAL closest to the beampipe. These cuts reduced the rate of background events while preserving high efficiency $(>99 \%$ ) for the signal events.

The following criteria were applied offline to reconstruct and select the events:

- the identification and energy measurement of the scattered electron used information from the CAL. The energy was required to satisfy $E>10 \mathrm{GeV}$. The impact point of the electron on the CAL was measured using three detectors: SRTD, HES and CAL. Given its superior position resolution, preference was given to the measurement from the SRTD. This was improved by the position obtained from HES or CAL when applicable. To ensure full containment of the electromagnetic shower and good position reconstruction, fiducial cuts were applied to the impact position of the electron on the face of the RCAL;

- the $\phi$ mesons were reconstructed from the properties of the decay kaons. No particle identification was used. Events with two tracks of opposite charge each with $p_{T}>$ $0.15 \mathrm{GeV}$ and $|\eta|<1.7$ were selected. These tracks were assigned the kaon mass

and the invariant mass was formed. Track combinations with invariant masses falling within an allowed mass window were selected (see below). Events with additional tracks not associated with the scattered electron or with kaon decays were rejected;

- the position of the reconstructed vertex was required to be compatible with that of an ep collision, $\left|Z_{\mathrm{VTX}}\right|<50 \mathrm{~cm}$. The radial distance of the reconstructed vertex from the nominal beamline was required to be less than $0.8 \mathrm{~cm}$ to remove $K_{S}^{0} \rightarrow \pi^{+} \pi^{-}$decays; 
- to remove events with large initial-state radiation, $45<E-P_{Z}<65 \mathrm{GeV}$ was imposed, where the longitudinal energy-momentum variable $E-P_{Z}$ is calculated using the momenta of the two kaons and the scattered electron (the masses are neglected). This variable is peaked at twice the electron beam energy $(55 \mathrm{GeV})$ for non-radiative DIS events. The $E-P_{Z}>45 \mathrm{GeV}$ cut removed events with a radiated photon of more than $5 \mathrm{GeV}$;

- to suppress non-exclusive events, the energy of each CAL cluster not associated with either of the final-state kaons or the scattered electron was required to be less than $0.3 \mathrm{GeV}$. To suppress further the contamination from proton-dissociative events, $e p \rightarrow$ e $\phi Y$, the energy in the FPC was required to be less than $1 \mathrm{GeV}$. These cuts restrict the mass of the proton-dissociative system, $Y$, to $M_{Y} \lesssim 2.3 \mathrm{GeV}$.

Events were required to be in a kinematic region where the properties of the final-state particles are well measured. Additionally, the kinematic range was limited to the region where the acceptance varies only slowly with the kinematic variables. This led to the following selection:

$$
\begin{aligned}
Q^{2} & >2 \mathrm{GeV}^{2}, \\
|t| & <0.6 \mathrm{GeV}^{2}, \\
33.75 \mathrm{GeV}+\left(1.25 \mathrm{GeV}^{-1} \cdot Q^{2}\right) & <W<100 \mathrm{GeV}+\left(3.7 \mathrm{GeV}^{-1} \cdot Q^{2}\right),
\end{aligned}
$$

with $Q^{2}$ given in $\mathrm{GeV}^{2}$ in the last expression. The distribution of selected events in the $x-Q^{2}$ plane for $1.01<m_{K K}<1.04 \mathrm{GeV}$ is shown in Fig. 1.

\section{Monte Carlo simulation}

The acceptance and the effects of the detector response were determined using samples of Monte Carlo (MC) events. All generated events were passed through the standard ZEUS detector simulation, based on the GEANT 3.13 program [39], the ZEUS trigger simulation package, and the ZEUS reconstruction software.

The exclusive process $e p \rightarrow e \phi p$ was modelled using the ZEusvm [40] MC generator interfaced to HERACLES 4.6.1 [41] to account for first-order QED radiative effects. The parameters describing the $W, Q^{2}, t$ and $\theta_{h}$ distributions in the MC simulation were adjusted such that the $\mathrm{MC}$ simulation reproduced the data distributions. The typical acceptance for $\phi \rightarrow K^{+} K^{-}$increases from $20 \%$ at $Q^{2}=2 \mathrm{GeV}^{2}$ to $60 \%$ for $Q^{2}>10 \mathrm{GeV}^{2}$. The acceptance is small at the smaller $Q^{2}$ due to the fiducial cuts on the impact point of the electron. 
Proton-dissociative events, ep $\rightarrow e \phi Y$, were modelled using the generator EPSOFT [42]. The $\gamma^{*} p \rightarrow \phi Y$ cross section was parametrized as

$$
\frac{d^{2} \sigma^{\gamma^{*} p \rightarrow \phi Y}}{d t d M_{Y}^{2}} \propto f\left(Q^{2}, W\right) e^{-b|t|} M_{Y}^{-\beta}
$$

with the same $Q^{2}$ and $W$ dependence, $f\left(Q^{2}, W\right)$, as for the exclusive case. The values of $b$ and $\beta$ are discussed in Section [7.2.

\section{$7 \quad$ Extraction of the $\phi$ signal}

Figure 2 shows the invariant-mass distribution of the $K^{+} K^{-}$pairs obtained after the selection described in Section 5. A total of $3642 \phi$ candidates were found after subtraction of non-resonant background in the mass range $1.01<m_{K K}<1.04 \mathrm{GeV}$. The natural width of the resonance is comparable to the detector mass resolution, which is $1.8 \mathrm{MeV}$ for tracks at central rapidity, deteriorating to $\sim 5 \mathrm{MeV}$ for tracks crossing the CTD through large angles. This resolution is well described by the MC simulation.

\subsection{Non-resonant background}

The non-resonant background was estimated for each bin in which a cross section was extracted with an unbinned likelihood fit to the invariant-mass distribution. The assumed functional form was a p-wave relativistic Breit-Wigner convoluted with a Gaussian resolution function for the signal plus a background function with the shape $a\left(m_{K K}-2 m_{K}\right)^{b}$. The background, estimated from the fit, was subtracted from the number of observed events in the mass window. The contribution of the non-resonant background in the mass range of the signal is typically $18 \%$ at $Q^{2}=2.4 \mathrm{GeV}^{2}$, decreasing to $5 \%$ at $Q^{2}=13 \mathrm{GeV}^{2}$.

\subsection{Proton-dissociative background}

The remaining source of background consists of $\phi$ production accompanied by proton dissociation, e $p \rightarrow e \phi Y$, where the particles from the breakup of the proton are not detected. A similar study to that done for $J / \psi$ production [12] was performed.

Proton-dissociative events with observed proton breakup were studied using a sample of diffractive events selected as described in Section 5, with the following exceptions:

- the elasticity criteria (last bullet in Section 5) were not applied and the energy in FPC was required to satisfy $E_{\mathrm{FPC}}>1 \mathrm{GeV}$; 
- a pseudorapidity gap $\Delta \eta>3$ was required between the energy deposits from the system $Y$ and the $\phi$ decay products.

The sample of proton-dissociation events identified with the FPC contained $300 \phi$ candidates for $|t|<1 \mathrm{GeV}^{2}$ in the kinematic range $45<W<135 \mathrm{GeV}$ and $Q^{2}>2 \mathrm{GeV}^{2}$. The $W, Q^{2}$ and $\theta_{h}$ dependences were found to be the same as for the elastic data sample. The $t$ dependence was found to have a slope (see Eq. 1) decreasing with $Q^{2}$ as $b=(5.1 \pm 1.3) /\left(1+R\left(Q^{2}\right)\right) \mathrm{GeV}^{-2}$ with $R$ given in Section 8.5.1. The MC distribution of $M_{Y}^{2}$ was tuned to describe the FPC energy distribution, yielding $\beta=2.0 \pm 0.5$.

The fraction of proton-dissociative events in the final sample, averaged over $t$ for $|t|<$ $0.6 \mathrm{GeV}^{2}$, was $f_{\mathrm{p}-\text { diss }}=\left(7.0 \pm 0.4_{-2.8}^{+4.2}\right) \%$, independent of $W, Q^{2}$ and $\theta_{h}$. The uncertainty is dominated by the uncertainty in the modelling of the $M_{Y}$ spectrum for $M_{Y}<3 \mathrm{GeV}$, and by the simulation of the proton-remnant final state. The uncertainty of the behavior of the $M_{Y}$ spectrum at small masses was parametrized by allowing $\beta$ to vary in the range $1.5<\beta<3.0$. The uncertainties on the simulation of the proton-remnant final state were estimated by a comparison of different MC simulations.

The fraction of proton-dissociative events increases from $4.5 \%$ for $0<|t|<0.08 \mathrm{GeV}^{2}$ to $14.5 \%$ for $0.35<|t|<0.6 \mathrm{GeV}^{2}$. The cross sections presented in the next sections were corrected for this background in bins of $t$, and globally in $W, Q^{2}$ and $\theta_{h}$.

\section{Results}

\subsection{Cross sections}

In each bin of a kinematic variable, the ep cross section was extracted using the formula

$$
\sigma^{e p \rightarrow e \phi p}=\frac{\left(N_{\text {data }}-N_{\mathrm{bgd}}\right)\left(1-f_{\mathrm{p}-\text { diss }}\right)}{\mathcal{A B} \mathcal{L}}
$$

where $N_{\text {data }}$ is the number of events in the data and $N_{\mathrm{bgd}}$ is the number of events from the non-resonant background. The overall acceptance is denoted as $\mathcal{A}, \mathcal{B}$ accounts for the $\phi \rightarrow K^{+} K^{-}$decay branching ratio (49.2 \pm 0.6$) \%$ [43], and $\mathcal{L}$ is the integrated luminosity.

The total exclusive photon-proton cross section was calculated as

$$
\sigma_{\mathrm{tot}}^{\gamma^{*} p \rightarrow \phi p}\left(Q^{2}, W, t\right)=\sigma_{\mathrm{tot}, \mathrm{MC}}^{\gamma^{*} p \rightarrow \phi p}\left(Q^{2}, W, t\right) \cdot \frac{\sigma_{\mathrm{DATA}}^{e p \rightarrow e \phi p}}{\sigma_{\mathrm{MC}}^{e p \rightarrow e \phi p}} .
$$

In this way, the MC simulation was used to correct for radiative effects, detector acceptance, cut efficiencies and the shape of the cross section within the bin. 
The cross sections were measured for $|t|<0.6 \mathrm{GeV}^{2}$. The results were then extrapolated to the full $t$ range assuming $d \sigma / d t \propto e^{-b|t|}$. The correction factor needed to extrapolate to the cross section integrated over $t$ was evaluated bin-by-bin using the measured value of $b$ for the given $Q^{2}$, and ranged from $1.5 \%$ to $5.0 \%$. The cross sections are quoted for the mass range $2 m_{K}<m_{K K}<M_{\phi}+5 \Gamma$, where $M_{\phi}=1019.4 \mathrm{MeV}$ and $\Gamma=4.458 \mathrm{MeV}$ [43].

\subsection{Systematic uncertainties}

The systematic uncertainties on the measured cross sections were determined by varying the selection cuts and by modifying the analysis procedure. The sources of systematic uncertainties considered [36] were similar to those of previously published analyses $[12,44]$. Except for the two lowest $Q^{2}$ bins in the evaluation of $d \sigma / d Q^{2}$, the systematic uncertainties (excluding normalization uncertainty) were smaller than the statistical errors.

- The electron position reconstruction is critical in determining the acceptance and in the kinematic-variable reconstruction. Possible misalignments of the SRTD and CAL were estimated to be $2 \mathrm{~mm}$, and the effect of such misalignments was tested via MC simulations. In addition, the electron fiducial cut was varied by $5 \mathrm{~mm}$. The incomplete knowledge of the reconstructed electron position generally provided the largest source of uncertainty.

- The elasticity cut is important in suppressing non-exclusive events. The energy distribution of the most energetic cluster not assigned to the electron or one of the kaons was compared in data and MC simulation. The MC simulation was found to reproduce the data distributions well, indicating a very small non-exclusive contribution with extra particles in the central region. The cut was varied from 0.3 to $0.4 \mathrm{GeV}$ in both data and MC simulation to determine the uncertainties.

- The stability of the fitting procedure and extraction of the signal was checked by reducing the fit range to $m_{K K}<1.07 \mathrm{GeV}$ and changing the accepted mass range to $1.01<m_{K K}<1.035 \mathrm{GeV}$, respectively. Except for bins with a small number of events the contribution to the overall uncertainty was small.

- Uncertainties from the dependence on the MC parametrizations were also estimated and found to be small compared to other sources of systematic uncertainties.

- The normalization uncertainty was different for different measurements. All measurements had a normalization uncertainty due to uncertainties in the integrated luminosity, $\pm 2.5 \%$, and due to the uncertainty in the decay branching ratio, $\pm 1.2 \%$, leading to a combined uncertainty of $\pm 2.8 \%$. In the case of the $d \sigma / d Q^{2}$ and $d \sigma / d W$ measurements, the proton-dissociation background was subtracted globally and the normalization uncertainty increased to ${ }_{-5.3}^{+4.1} \%$. The uncertainty due to the subtraction 
of proton-dissociative background for the $d \sigma / d t$ measurement resulted in a $t$-dependent uncertainty as discussed in Section [7.2] For the angular distributions, the normalization uncertainty does not appear as only the shapes of the distributions were measured.

The total systematic uncertainty (excluding the normalization uncertainty) was determined by adding the individual contributions in quadrature. The correlated and uncorrelated systematic uncertainties were evaluated separately. The typical size of the systematic uncertainty (excluding the normalization uncertainty) was $5 \%$ for the cross sections in $W$ and $Q^{2}$ bins, and $5 \%$ at small $|t|$, increasing to $10 \%$ at $|t|=0.6 \mathrm{GeV}^{2}$.

\subsection{Dependence on $W$ and $Q^{2}$}

The cross-section $\sigma_{\text {tot }}^{\gamma^{*} p \rightarrow \phi p}$, measured as a function of $W$ and $Q^{2}$, is shown in Fig. Ba and given in Table 1 In addition to the measured cross section, this table gives the kinematic range over which the measurement was performed, the value of the kinematic variables at which the cross section is quoted, the acceptance, and the background-corrected number of $\phi$ events. The much higher statistics available for this analysis allow measurements of double-differential cross sections.

The cross sections were fitted to a dependence $\sigma \propto W^{\delta}$ with results presented in Table 2 , The measured values of $\delta$ show no $Q^{2}$ dependence within the present uncertainties. The values of $\delta$ are compared to those from previous H1 [4] and ZEUS $[1,2,12,13]$ measurements in Fig. B $\mathrm{b}$, where the data are plotted as a function of $Q^{2}+M_{V}^{2}$. The values of $\delta$ scale with this variable within the present uncertainties.

The $Q^{2}$ dependence of the cross section, given in Table [3, is shown in Fig. [4 for $W=$ $75 \mathrm{GeV}$. The data are compared to previous ZEUS $[8,9]$ and $\mathrm{H} 1[10]$ results. The $\phi$ data from this analysis were fitted with a function of the form $\sigma \propto\left(Q^{2}+M_{\phi}^{2}\right)^{-n}$ expected in the VDM [45]. The new high-precision data show that the $Q^{2}$ dependence of the cross section cannot be fitted with a single value of $n$ over the $Q^{2}$ range of this analysis. The fit parameter $n$ was found to vary from $n=2.087 \pm 0.055$ (stat.) \pm 0.050 (syst.) for $2.4 \leq Q^{2} \leq 9.2 \mathrm{GeV}^{2}$ to $n=2.75 \pm 0.13$ (stat.) \pm 0.07 (syst.) for $9.2 \leq Q^{2} \leq 70 \mathrm{GeV}^{2}$. There are several possible causes that could lead to this behavior, including a variation of $R$ with $Q^{2}$, the dependence of $\alpha_{S}$ on $Q^{2}$ and the changing $Q^{2}$ dependence of the gluon density at fixed $W$. The longitudinal and transverse components of the cross section have been separately extracted using the measured value of $R$ (see Section 8.5.1), and are shown

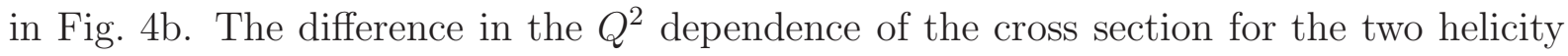
components is clearly seen. 


\subsubsection{Comparison to Models}

The predictions of the MRT model [26] were compared to the data using three different gluon densities as shown in Fig. 5 . The predicted $W$ dependence with the ZEUS-S [46] and CTEQ6M [47] gluon densities are compatible with the data, whereas the predictions using the MRST99 [48] gluon density are too steep. The predicted $Q^{2}$ dependence is too steep for all gluon densities.

The FS04 prediction [24] is also compared to the data in Fig. [5. The $W$ dependence seen in the data is well reproduced, although the normalization is somewhat low at large $Q^{2}$ and high at low $Q^{2}$. A similar model [49] which did not employ a saturated dipole cross section showed a somewhat steeper $W$ dependence.

The expectations from the MRT and FS04 models for the $Q^{2}$ dependence are also compared to the data in Fig. 囵. In this case, the ZEUS-S gluon density was chosen for the MRT model. This prediction agrees reasonably well with the data at higher $Q^{2}$. At small $Q^{2}$, the predicted transverse cross section is too high while the predicted longitudinal cross section is too low. The FS04 prediction reproduces the data better than the MRT prediction for the longitudinal component, and is very similar to the MRT prediction for the transverse component.

\subsection{Dependence on $t$}

The differential cross section, $d \sigma_{\mathrm{tot}}^{\gamma^{*} p \rightarrow \phi p} / d|t|$, measured as a function of $t$ in the range $|t|<0.6 \mathrm{GeV}^{2}$, is shown in Fig. 6a for different values of $Q^{2}$ and $W=75 \mathrm{GeV}$. A function of the form $d \sigma / d t=d \sigma /\left.d t\right|_{t=0} \cdot e^{b t}$ was fitted to the data and the results of the fit are given in Fig. [6 b along with measurements from other vector mesons $[1,2,4,8,10,12,13]$. The slope parameters from this analysis are given in Table 6 . The values of $b$ from this analysis show no $Q^{2}$ dependence within the present measurement uncertainties. The measurements in Fig. [6 b are presented as a function of $Q^{2}+M_{V}^{2}$ and are found to scale with this variable within the present data uncertainties.

The data sample was analyzed to determine the $W$ dependence as a function of $t$. In the Regge formalism, the differential cross section can be expressed as

$$
d \sigma / d t \propto W^{4\left(\alpha_{\mathbb{P}}(t)-1\right)},
$$

where $\alpha_{\mathbb{P}}$, the Pomeron trajectory, is usually parametrized as

$$
\alpha_{\mathbb{P}}(t)=\alpha_{\mathbb{P}}(0)+\alpha_{\mathbb{P}}^{\prime} t .
$$

The trajectory measured in soft diffractive processes is $\alpha_{\mathbb{P}}=1.08+0.25 t[22,23]$. In contrast, $\alpha_{\mathbb{P}}^{\prime}$ is much smaller in $J / \psi$ photoproduction: $\alpha_{\mathbb{P}}=1.20+0.115 t$ [12]. In 
this notation, $\alpha_{\mathbb{P}}=1+\delta / 4$. The values of $\alpha_{\mathbb{P}}(0)$ and $\alpha_{\mathbb{P}}^{\prime}$ were determined by fitting the $W$ dependence of the differential cross section at different $|t|$ values using Eq. (2) for $Q^{2}=5 \mathrm{GeV}^{2}$. Since the proton-dissociative process was found to have the same $W$ dependence as the exclusive process, the extraction of $\alpha_{\mathbb{P}}$ is not sensitive to this background contribution. The analysis was therefore extended up to $|t|=1 \mathrm{GeV}^{2}$. The fit results are shown in Fig. $7 \mathrm{~h}$ and are given in Table 5. The parameters of the trajectory were determined from a fit of Eq.(3) to the extracted $\alpha_{\mathbb{P}}(t)$ values, as shown in Fig. [7b.

The results are:

$$
\begin{aligned}
& \alpha_{\mathbb{P}}(0)=1.10 \pm 0.02 \text { (stat.) } \pm 0.02 \text { (syst.) } \\
& \alpha_{\mathbb{P}}^{\prime}=0.08 \pm 0.09 \text { (stat.) } \pm 0.08 \text { (syst.) } \mathrm{GeV}^{-2}
\end{aligned}
$$

The value of $\alpha_{I P}^{\prime}$ is closer to the value measured in $J / \psi$ production than that measured in soft diffractive processes.

\subsection{Decay angular distributions}

The angular distributions of the decay of the $\phi$ provide information about the photon and $\phi$ polarization states. In the helicity frame [50], the production and decay of the $\phi$ can be described in terms of three angles: $\Phi_{h}$, the angle between the $\phi$ production plane and the lepton scattering plane; $\theta_{h}$, the polar angle, and $\phi_{h}$, the azimuthal angle of the positively charged kaon. Under the assumption of $s$-channel helicity conservation (SCHC), the normalized angular distribution depends only on two angles, $\theta_{h}$ and $\psi_{h}=\phi_{h}-\Phi_{h}$. The $\theta_{h}$ distribution can be expressed in the form

$$
\frac{1}{N} \frac{d N}{d \cos \theta_{h}}=\frac{3}{8}\left[1+r_{00}^{04}+\left(1-3 r_{00}^{04}\right) \cos ^{2} \theta_{h}\right]
$$

The spin-density matrix-element $r_{00}^{04}$ represents the probability that the $\phi$ is produced in the helicity-0 state from a virtual photon of helicity 0 or 1.

The normalized cross section $\frac{1}{\sigma} \frac{d \sigma_{\text {tot }}^{\gamma^{*} p \rightarrow \phi p}}{d \cos \left(\theta_{h}\right)}$ at $W=90 \mathrm{GeV}$ is shown in Fig. 8 for seven values of $Q^{2}$. The data were fitted to Eq. (4). The values of $r_{00}^{04}$, determined from the fits, are given in Table 6] and plotted as a function of $Q^{2} / M_{V}^{2}$ in Fig. 9, where they are compared to the values extracted for other vector mesons $[1-3,8,9,12,13]$. The values of $r_{00}^{04}$ for the different vector mesons are found to scale in this variable.

The values of $r_{00}^{04}$ were also extracted as a function of $W$ for two different $Q^{2}$ values, and these are given in Table 7 . No dependence on $W$ was observed. 


\subsubsection{Longitudinal and transverse cross sections}

The ratio of the longitudinal to transverse cross section, $R=\sigma_{L} / \sigma_{T}$, was calculated as a function of $Q^{2}$ from $r_{00}^{04}$ according to the relation

$$
R=\frac{1}{\epsilon} \frac{r_{00}^{04}}{1-r_{00}^{04}}
$$

which is valid under the assumption of $\mathrm{SCHC}^{3}$. The average value of $\epsilon=0.99$ was used in extracting $R$. The values of $R$ are presented as a function of $Q^{2}$ in Table [ and as a function of $W$ in Table 7 . The scaling of $r_{00}^{04}$ with $Q^{2} / M_{V}^{2}$ implies that $R$ also scales in this variable. This is expected in dipole models of diffraction [51].

The values of $R$ are compared with previous ZEUS [8,9] and H1 [10] results in Fig. [10] as a function of $Q^{2}$ and in Fig. 10p as a function of $W$. The $Q^{2}$ dependence is well described by the expression $R=a\left(Q^{2} / M_{\phi}^{2}\right)^{b}$. The parameters were extracted from the fit to $r_{00}^{04}$ data for which the statistical errors are Gaussian, yielding $a=0.51 \pm 0.07$ (stat.) \pm 0.05 (syst.) and $b=0.86 \pm 0.11$ (stat.) \pm 0.05 (syst.). The prediction from the MRT model (using the ZEUS-S gluon density) is also shown in Fig. 10, as is the FS04 prediction. The general power-law dependence of $R$ with $Q^{2}$ is reproduced, but the model predictions systematically underestimate the measurements. The dipole-model prediction (FS04) is in somewhat better agreement with the ZEUS data than the MRT-model prediction (with the ZEUS-S gluon density), particularly at the lower $Q^{2}$ values. The weak dependence of $R$ on $W$ observed in Fig. 10p is consistent with both the MRT and FS04 models.

\section{Summary}

The exclusive electroproduction of $\phi$ mesons, $e p \rightarrow e \phi p$, has been measured with the ZEUS detector at HERA for photon virtualities in the range $2<Q^{2}<70 \mathrm{GeV}^{2}$, for photon-proton center-of-mass energies in the range $35<W<145 \mathrm{GeV}$ and for fourmomentum-transfer squared in the range $|t|<0.6 \mathrm{GeV}^{2}$. The extracted $\gamma^{*} p$ cross section rises with $W$ as $\sigma \propto W^{\delta}$, with a slope parameter $\delta \approx 0.4$. This value is between the 'soft' diffraction value and the value observed in $J / \psi$ production. No $Q^{2}$ or $t$ dependence of $\delta$ was observed within the present experimental precision of the data.

The high-precision data from this analysis reveal that the $Q^{2}$ dependence of the cross section cannot be fitted with a single power over the measured $Q^{2}$ range. The longitudinal and transverse components of the cross section were separately extracted using the

\footnotetext{
${ }^{3}$ The validity of SCHC has been tested for other vector mesons and found to be adequate for the purpose of extracting $R$ [3].
} 
measured value of $R$ and the different $Q^{2}$ dependence of the cross section for the two helicity components is clearly seen.

The $t$ distribution, measured for $|t|<0.6 \mathrm{GeV}^{2}$, is well described by an exponential dependence over the range $2<Q^{2}<70 \mathrm{GeV}^{2}$. The slope ranges from $6.4 \pm 0.4 \mathrm{GeV}^{-2}$ at $Q^{2}=2.4 \mathrm{GeV}^{2}$ to $5.1 \pm 1.1 \mathrm{GeV}^{-2}$ at $Q^{2}=19.7 \mathrm{GeV}^{2}$.

The ratio of the cross sections for longitudinally and transversely polarized photons, $R$, increases with $Q^{2}$ and can be fitted by a power-law dependence.

The data from this analysis were compared to previously published $e p \rightarrow e V p$ data. The values of $\delta$ and $b$ were found to scale, within the present accuracy, when plotted as a function of $Q^{2}+M_{V}^{2}$. The ratio of longitudinal to transverse cross sections was seen to scale with $Q^{2} / M_{V}^{2}$.

The MRT model does not reproduce the $Q^{2}$ dependence of the $\gamma^{*} p$ cross section observed in the data, while the FS04 prediction reasonably reproduces the data for the longitudinal photon polarization. This conclusion is also reflected in the better agreement of FS04 with the data for the measurement of $R$ vs $Q^{2}$. The $W$ dependence of the data can be reproduced in both models.

\section{Acknowledgements}

We thank the DESY directorate for their strong support and encouragement. The special effort of the HERA group is gratefully acknowledged. The design, construction and installation of the ZEUS detector has been made possible by the efforts of many people who are not listed as authors. Special thanks go to Thomas Teubner for providing us with the predictions from the MRT model, and to Jeff Forshaw for providing us with the predictions from the FS model. 


\section{References}

[1] ZEUS Coll., J. Breitweg et al., Eur. Phys. J. C 2 (1998) 247.

[2] ZEUS Coll., J. Breitweg et al., Eur. Phys. J. C 6 (1999) 603.

[3] ZEUS Coll., J. Breitweg et al., Eur. Phys. J. C 12 (2000) 393.

[4] H1 Coll., C. Adloff et al., Eur. Phys. J. C 13 (2000) 371.

[5] H1 Coll., C. Adloff et al., Phys. Lett. B 539 (2002) 25.

[6] ZEUS Coll., M. Derrick et al., Z. Phys. C 73 (1996) 73.

[7] ZEUS Coll., J. Breitweg et al., Phys. Lett. B 487 (2000) 273.

[8] ZEUS Coll., M. Derrick et al., Phys. Lett. B 377 (1996) 259.

[9] ZEUS Coll., M. Derrick et al., Phys. Lett. B 380 (1996) 220.

[10] H1 Coll., C. Adloff et al., Phys. Lett. B 483 (2000) 360.

[11] H1 Coll., C. Adloff et al., Eur. Phys. J. C 10 (1999) 373.

[12] ZEUS Coll., S. Chekanov et al., Eur. Phys. J. C 24 (2002) 345.

[13] ZEUS Coll., S. Chekanov et al., Nucl. Phys. B 695 (2004) 3.

[14] H1 Coll., C. Adloff et al., Phys. Lett. B 541 (2002) 251.

[15] ZEUS Coll., J. Breitweg et al., Phys. Lett. B 437 (1998) 432.

[16] H1 Coll., C. Adloff et al., Phys. Lett. B 483 (2000) 23.

[17] B.Z. Kopeliovich, et al., Phys. Lett. B 324 (1994) 469.

[18] S.J. Brodsky et al., Phys. Rev. D 50 (1994) 3134.

[19] P.D.B. Collins, An Introduction to Regge Theory and High Energy Physics. Cambridge University Press, 1977.

[20] M.G. Ryskin, Sov. J. Nucl. Phys. 52 (1990) 529.

[21] M.G. Ryskin, Z. Phys. C 57 (1993) 89.

[22] A. Donnachie and P.V. Landshoff, Phys. Lett. B 348 (1995) 213.

[23] G.A. Jaroszkiewicz and P.V. Landshoff, Phys. Rev. D 10 (1974) 170.

[24] J.R. Forshaw and G. Shaw, Preprint hep-ph/0411337, 2004.

[25] J. Forshaw, private communication.

[26] A.D. Martin, M.G. Ryskin and T. Teubner, Phys. Rev. D 62 (2000) 14022.

[27] I.P. Ivanov, N.N. Nikolaev, A.A. Savin, Preprint hep-ph/0501034, 2005. 
[28] ZEUS Coll., U. Holm (ed.), The ZEUS Detector. Status Report (unpublished), DESY (1993), available on http://www-zeus.desy.de/bluebook/bluebook.html.

[29] N. Harnew et al., Nucl. Instr. Meth. A 279 (1989) 290;

B. Foster et al., Nucl. Phys. Proc. Suppl. B 32 (1993) 181;

B. Foster et al., Nucl. Instr. Meth. A 338 (1994) 254.

[30] M. Derrick et al., Nucl. Instr. Meth. A 309 (1991) 77;

A. Andresen et al., Nucl. Instr. Meth. A 309 (1991) 101;

A. Caldwell et al., Nucl. Instr. Meth. A 321 (1992) 356;

A. Bernstein et al., Nucl. Instr. Meth. A 336 (1993) 23.

[31] A. Bamberger et al., Nucl. Instr. Meth. A 450 (2000) 235.

[32] A. Bamberger et al., Nucl. Instr. Meth. A 401 (1997) 63.

[33] A. Dwurazny et al., Nucl. Instr. Meth. A 277 (1989) 176.

[34] J. Andruszków et al., Acta Phys. Pol. B 32 (2001) 2025.

[35] L.N. Hand, Phys. Rev. 129 (1963) 1834.

[36] M. Helbich. Ph.D. Thesis, Columbia University, 2004, unpublished.

[37] W.H. Smith, K. Tokoshuku and L.W. Wiggers, Proc. Computing in High-Energy Physics (CHEP), Annecy, France, C. Verkerk and W. Wojcik (eds.), p. 222. (1992). Also in preprint DESY 92-150B.

[38] W.H. Smith et al., Nucl. Instr. Meth. A 355 (1995) 278.

[39] R. Brun et al., GEAnt3, Technical Report CERN-DD/EE/84-1, CERN, 1987.

[40] K. Muchorowski. Ph.D. Thesis, Warsaw University, Warsaw, Poland, 1996, unpublished.

[41] A. Kwiatkowski, H. Spiesberger and H.-J. Möhring, Comput. Phys. Comm. 69 (1992) 155. Also in Proc. Workshop Physics at HERA, W. Buchmüller and G.Ingelman (eds.), DESY, Hamburg, (1991);

H. Spiesberger, An Event Generator for ep Interactions at HERA Including Radiative Processes (Version 4.6), 1996, available on

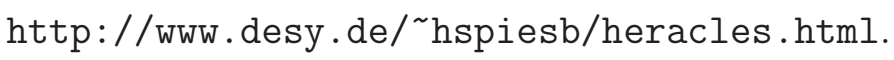

[42] L. Adamczyk. Ph.D. Thesis, University of Mining and Metallurgy, Cracow, Poland, Report DESY-THESIS-1999-045, DESY, 1999;

M. Kasprzak. Ph.D. Thesis, Warsaw University, Warsaw, Poland, Report DESY F35D-96-16, DESY, 1996.

[43] Particle Data Group, K. Hagiwara et al., Phys. Rev. D 66 (2002) 31.

[44] ZEUS Coll., S. Chekanov et al., Eur. Phys. J. C 21 (2001) 443. 
[45] J.J. Sakurai, Phys. Rev. Lett. 22 (1969) 981.

[46] ZEUS Coll., S. Chekanov et al., Phys. Rev. D 67 (2003) 12007.

[47] J. Pumplin, et al., JHEP 0207 (2002) 012.

[48] A.D. Martin, et al., Eur. Phys. J. C 14 (2000) 133.

[49] J.R. Forshaw, G. Kerley and G. Shaw, Phys. Rev. D 60 (1999) 074012.

[50] K. Schilling and G. Wolf, Nucl. Phys. B 61 (1973) 381.

[51] V. Barone and E. Predazzi, High Energy Particle Diffraction, in Texts and Monographs in Physics. Springer Verlag, Berlin (Germany), 2002. 


\begin{tabular}{|c|c|c|c|c|c|c|}
\hline $\begin{array}{c}Q^{2} \text { range } \\
\left(\mathrm{GeV}^{2}\right)\end{array}$ & $\begin{array}{c}Q^{2} \\
\left(\mathrm{GeV}^{2}\right) \\
\end{array}$ & $\begin{array}{c}W \text { range } \\
(\mathrm{GeV})\end{array}$ & $\begin{array}{c}W \\
(\mathrm{GeV})\end{array}$ & Events & $\begin{array}{c}A \\
(\%)\end{array}$ & $\begin{array}{c}\sigma_{\text {tot }}^{\gamma^{*} p \rightarrow \phi p} \\
(\mathrm{nb})\end{array}$ \\
\hline \multirow{7}{*}{$2-3$} & \multirow{7}{*}{2.4} & $35-45$ & 40 & 203 & 19.4 & $76.4 \pm 6.5_{-5.9}^{+4.0}$ \\
\hline & & $45-55$ & 50 & 255 & 18.7 & $101.2 \pm 7.7_{-4.2}^{+8.1}$ \\
\hline & & $55-65$ & 60 & 220 & 19.6 & $101.9 \pm 8.3_{-7.4}^{+6.3}$ \\
\hline & & $65-75$ & 70 & 210 & 19.6 & $112.8 \pm 9.4_{-7.6}^{+6.3}$ \\
\hline & & $75-85$ & 80 & 167 & 19.3 & $107 \pm 11_{-6}^{+4}$ \\
\hline & & $85-95$ & 90 & 175 & 20.7 & $122 \pm 11_{-10}^{+10}$ \\
\hline & & $95-105$ & 100 & 134 & 19.5 & $110 \pm 11_{-7}^{+8}$ \\
\hline \multirow{6}{*}{$3-5$} & \multirow{6}{*}{3.8} & $40-50$ & 45 & 184 & 20.9 & $47.0 \pm 4.1_{-2.5}^{+1.4}$ \\
\hline & & $50-60$ & 55 & 146 & 21.3 & $44.3 \pm 4.5_{-3.6}^{+2.8}$ \\
\hline & & $60-70$ & 65 & 169 & 22.3 & $56.7 \pm 5.1_{-4.0}^{+3.6}$ \\
\hline & & $70-85$ & 77.5 & 218 & 22.0 & $62.3 \pm 5.0_{-4.2}^{+4.3}$ \\
\hline & & $85-100$ & 92.5 & 158 & 21.3 & $57.4 \pm 5.4_{-4.3}^{+3.2}$ \\
\hline & & $100-115$ & 107.5 & 123 & 21.2 & $59.0 \pm 6.2_{-2.6}^{+3.0}$ \\
\hline \multirow{6}{*}{$5-9$} & \multirow{6}{*}{6.5} & $45-55$ & 50 & 111 & 34.6 & $16.4 \pm 1.8_{-1.1}^{+1.1}$ \\
\hline & & $55-70$ & 62.5 & 168 & 37.6 & $19.1 \pm 1.7_{-1.4}^{+1.5}$ \\
\hline & & $70-85$ & 77.5 & 136 & 38.5 & $19.6 \pm 1.9_{-1.5}^{+1.6}$ \\
\hline & & $85-100$ & 92.5 & 123 & 37.8 & $21.6 \pm 2.3_{-1.1}^{+1.1}$ \\
\hline & & $100-115$ & 107.5 & 116 & 40.9 & $23.1 \pm 2.5_{-1.3}^{+1.3}$ \\
\hline & & $115-135$ & 125 & 70 & 44.6 & $25.3 \pm 3.5_{-3.9}^{+3.9}$ \\
\hline \multirow{6}{*}{$9-30$} & \multirow{6}{*}{13.0} & $50-60$ & 55 & 64.5 & 58.6 & $5.05 \pm 0.73_{-0.37}^{+0.37}$ \\
\hline & & $60-75$ & 67.5 & 88.6 & 57.0 & $4.96 \pm 0.59_{-0.26}^{+0.27}$ \\
\hline & & $75-90$ & 82.5 & 87.3 & 56.5 & $6.12 \pm 0.81_{-0.40}^{+0.34}$ \\
\hline & & $90-105$ & 97.5 & 86.6 & 55.6 & $6.82 \pm 0.85_{-0.37}^{+0.40}$ \\
\hline & & $105-125$ & 115 & 85.8 & 58.0 & $6.33 \pm 0.78_{-0.22}^{+0.50}$ \\
\hline & & $125-145$ & 135 & 61.7 & 56.8 & $6.65 \pm 0.97_{-0.52}^{+0.44}$ \\
\hline
\end{tabular}

Table 1: The $\gamma^{*} p \rightarrow \phi p$ cross section, $\sigma_{\text {tot }}^{\gamma^{*} p \rightarrow \phi p}$, as a function of $W$ and $Q^{2}$. The first uncertainty is statistical and the second systematic. The overall normalization error of ${ }_{-5.3}^{+4.1} \%$ is not included. The $Q^{2}$ and $W$ values at which the cross section is evaluated are given in the second and fourth columns. The number of extracted events corrected for non-resonant background and the acceptance, A, are given for each measurement. 


\begin{tabular}{|c|c|c|}
\hline $\begin{array}{c}Q^{2} \text { range } \\
\left(\mathrm{GeV}^{2}\right)\end{array}$ & $\begin{array}{c}Q^{2} \\
\left(\mathrm{GeV}^{2}\right)\end{array}$ & $\begin{array}{c}\delta \\
\left(\sigma \propto W^{\delta}\right)\end{array}$ \\
\hline $2-3$ & 2.4 & $0.41 \pm 0.10_{-0.05}^{+0.06}$ \\
$3-5$ & 3.8 & $0.34 \pm 0.13_{-0.03}^{+0.05}$ \\
$5-9$ & 6.5 & $0.43 \pm 0.15_{-0.13}^{+0.13}$ \\
$9-30$ & 13.0 & $0.38 \pm 0.18_{-0.06}^{+0.07}$ \\
\hline
\end{tabular}

Table 2: The $\delta$ parameter $\left(\sigma \propto W^{\delta}\right)$ as a function of $Q^{2}$. The first uncertainty is statistical and the second systematic.

\begin{tabular}{|c|c|c|c|c|}
\hline $\begin{array}{c}Q^{2} \text { range } \\
\left(\mathrm{GeV}^{2}\right)\end{array}$ & $\begin{array}{c}Q^{2} \\
\left(\mathrm{GeV}^{2}\right)\end{array}$ & Events & $\begin{array}{c}A \\
(\%)\end{array}$ & $\begin{array}{c}\sigma_{\text {tot }}^{\gamma^{*} p \rightarrow \phi p} \\
(\mathrm{nb})\end{array}$ \\
\hline $2-3$ & 2.4 & 1389 & 19.3 & $105.5 \pm 3.4_{-6.0}^{+4.6}$ \\
$3-4.5$ & 3.6 & 842 & 21.4 & $57.6 \pm 2.4_{-3.5}^{+3.2}$ \\
$4.5-6$ & 5.2 & 420 & 26.6 & $31.1 \pm 1.8_{-1.8}^{+1.7}$ \\
$6-8$ & 6.9 & 376 & 42.5 & $17.9 \pm 1.1_{-1.0}^{+1.0}$ \\
$8-11$ & 9.2 & 314 & 52.1 & $11.06 \pm 0.73_{-0.51}^{+0.56}$ \\
$11-15$ & 12.6 & 200 & 59.9 & $6.42 \pm 0.52_{-0.18}^{+0.24}$ \\
$15-20$ & 17.1 & 61.8 & 55.2 & $2.50 \pm 0.37_{-0.22}^{+0.16}$ \\
$20-30$ & 24.0 & 32.3 & 62.5 & $0.98 \pm 0.19_{-0.05}^{+0.05}$ \\
$30-70$ & 38.8 & 10.5 & 53.5 & $0.37 \pm 0.13_{-0.04}^{+0.04}$ \\
\hline
\end{tabular}

Table 3: The $\gamma^{*} p \rightarrow \phi p$ cross section, $\sigma_{\text {tot }}^{\gamma^{*} p \rightarrow \phi p}$, as a function of $Q^{2}$ for $W=$ $75 \mathrm{GeV}$. The second column gives the value of $Q^{2}$ at which the cross section is quoted. The number of extracted events corrected for non-resonant background and the acceptance, $A$, are given for each measurement. The first uncertainty is statistical and the second systematic. The overall normalization error of ${ }_{-5.3}^{+4.1} \%$ is not included. 


\begin{tabular}{|c|c|c|c|}
\hline $\begin{array}{c}Q^{2} \text { range } \\
\left(\mathrm{GeV}^{2}\right)\end{array}$ & $\begin{array}{c}Q^{2} \\
\left(\mathrm{GeV}^{2}\right)\end{array}$ & $\begin{array}{c}b\left(\frac{d \sigma}{d|t|} \propto e^{-b|t|}\right) \\
\left(\mathrm{GeV}^{-2}\right)\end{array}$ & $\begin{array}{c}\frac{d \sigma}{d|t|} \\
\left(\mathrm{nb} / \mathrm{GeV}^{2}\right)\end{array}$ \\
\hline $2-3$ & 2.4 & $6.37 \pm 0.32_{-0.28}^{+0.27}$ & $678 \pm 37_{-56}^{+39}$ \\
$3-4.5$ & 3.6 & $6.29 \pm 0.42_{-0.32}^{+0.45}$ & $352 \pm 26_{-22}^{+27}$ \\
$4.5-6$ & 5.2 & $5.26 \pm 0.48_{-0.37}^{+0.28}$ & $162 \pm 16_{-13}^{+9}$ \\
$6-8$ & 6.9 & $5.49 \pm 0.52_{-0.47}^{+0.33}$ & $98 \pm 10_{-10}^{+8}$ \\
$8-11$ & 9.2 & $5.58 \pm 0.54_{-0.32}^{+0.45}$ & $61.3 \pm 6.6_{-3.7}^{+4.8}$ \\
$11-15$ & 12.6 & $5.45 \pm 0.78_{-0.37}^{+0.21}$ & $32.9 \pm 5.5_{-3.2}^{+1.6}$ \\
$15-30$ & 19.7 & $5.10 \pm 0.98_{-0.40}^{+0.43}$ & $8.8 \pm 1.9_{-1.0}^{+0.8}$ \\
\hline
\end{tabular}

Table 4: The slope parameter, $b$, as a function of $Q^{2}$ for $W=75 \mathrm{GeV}$. The cross section is quoted at the $Q^{2}$ value given in the second column. The first uncertainty is statistical and the second systematic.

\begin{tabular}{|c|c|c|c|}
\hline $\begin{array}{c}|t| \text { range } \\
\left(\mathrm{GeV}^{2}\right)\end{array}$ & $\begin{array}{c}|t| \\
\left(\mathrm{GeV}^{2}\right)\end{array}$ & $\begin{array}{c}\delta \\
\left(\sigma \propto W^{\delta}\right)\end{array}$ & $\begin{array}{c}\alpha_{I P} \\
\alpha_{I P}=1+\delta / 4\end{array}$ \\
\hline $0-0.08$ & 0.025 & $0.34 \pm 0.11_{-0.08}^{+0.08}$ & $1.085 \pm 0.027_{-0.020}^{+0.021}$ \\
$0.08-0.20$ & 0.12 & $0.39 \pm 0.12_{-0.08}^{+0.09}$ & $1.098 \pm 0.030_{-0.021}^{+0.022}$ \\
$0.20-0.35$ & 0.25 & $0.44 \pm 0.15_{-0.11}^{+0.11}$ & $1.110 \pm 0.038_{-0.028}^{+0.027}$ \\
$0.35-0.6$ & 0.45 & $0.31 \pm 0.18_{-0.34}^{+0.26}$ & $1.078 \pm 0.045_{-0.086}^{+0.064}$ \\
$0.6-1.0$ & 0.73 & $-0.11 \pm 0.33_{-0.32}^{+0.24}$ & $0.972 \pm 0.083_{-0.080}^{+0.060}$ \\
\hline
\end{tabular}

Table 5: The $\delta$ parameter and the Pomeron trajectory $\alpha_{I P}$ as a function of $|t|$. The first uncertainty is statistical and the second systematic.

\begin{tabular}{|c|c|c|c|}
\hline $\begin{array}{c}Q^{2} \text { range } \\
\left(\mathrm{GeV}^{2}\right)\end{array}$ & $\begin{array}{c}Q^{2} \\
\left(\mathrm{GeV}^{2}\right)\end{array}$ & $R=\sigma_{L} / \sigma_{T}$ \\
\hline $2-3$ & 2.4 & $0.529 \pm 0.025_{-0.029}^{+0.025}$ & $1.13_{-0.11}^{+0.12}{ }_{-0.12}^{+0.12}$ \\
$3-4.5$ & 3.6 & $0.595 \pm 0.031_{-0.030}^{+0.036}$ & $1.49_{-0.18}^{+0.20}{ }_{-0.17}^{+0.24}$ \\
$4.5-6$ & 5.2 & $0.680 \pm 0.038_{-0.031}^{+0.026}$ & $2.15_{-0.33}^{+0.42}{ }_{-0.28}^{+0.28}$ \\
$6-8$ & 6.9 & $0.740 \pm 0.038_{-0.027}^{+0.028}$ & $2.88_{-0.50}^{+0.67}{ }_{-0.37}^{+0.47}$ \\
$8-11$ & 9.2 & $0.744 \pm 0.043_{-0.019}^{+0.017}$ & $2.94_{-0.57}^{+0.80}{ }_{-0.27}^{+0.27}$ \\
$11-15$ & 12.6 & $0.802 \pm 0.043_{-0.018}^{+0.022}$ & $4.09_{-0.91}^{+1.41}{ }_{-0.42}^{+0.63}$ \\
$15-30$ & 19.7 & $0.825 \pm 0.066_{-0.024}^{+0.019}$ & $4.77_{-1.58}^{+3.49}{ }_{-0.71}^{+0.72}$ \\
\hline
\end{tabular}

Table 6: The spin-density matrix-element $r_{00}^{04}$ and the ratio of cross sections for longitudinally and transversly polarized photons, $R$, as a function of $Q^{2}$. The first uncertainty is statistical and the second systematic. Due to the transformation given in Eq. (5), the error on the measurement of $R$ is asymmetric. 


\begin{tabular}{|c|c|c|c|c|c|}
\hline $\begin{array}{c}Q^{2} \text { range } \\
\left(\mathrm{GeV}^{2}\right)\end{array}$ & $\begin{array}{c}Q^{2} \\
\left(\mathrm{GeV}^{2}\right)\end{array}$ & $\begin{array}{c}W \text { range } \\
(\mathrm{GeV})\end{array}$ & $\begin{array}{c}W \\
(\mathrm{GeV})\end{array}$ & $r_{00}^{04}$ & $R=\sigma_{L} / \sigma_{T}$ \\
\hline \multirow{5}{*}{$2-5$} & \multirow{5}{*}{3} & $35-50$ & 42.5 & $0.597 \pm 0.038_{-0.019}^{+0.019}$ & $1.50_{-0.22}^{+0.26}{ }_{-0.12}^{+0.12}$ \\
\hline & & $50-65$ & 57.25 & $0.569 \pm 0.038_{-0.017}^{+0.017}$ & $1.33_{-0.19}^{+0.22}{ }_{-0.09}^{+0.10}$ \\
\hline & & $65-80$ & 72.25 & $0.511 \pm 0.041_{-0.020}^{+0.017}$ & $1.06_{-0.16}^{+0.19}{ }_{-0.08}^{+0.08}$ \\
\hline & & $80-95$ & 87.25 & $0.611 \pm 0.043_{-0.028}^{+0.026}$ & $1.59_{-0.26}^{+0.32}{ }_{-0.18}^{+0.19}$ \\
\hline & & $95-115$ & 102.75 & $0.612 \pm 0.048_{-0.033}^{+0.033}$ & $1.59_{-0.29}^{+0.37}{ }_{-0.21}^{+0.24}$ \\
\hline \multirow{5}{*}{$5-20$} & \multirow{5}{*}{8} & $45-60$ & 52.25 & $0.800 \pm 0.042_{-0.029}^{+0.031}$ & $4.05_{-0.87}^{+1.33}{ }_{-0.64}^{+0.93}$ \\
\hline & & $60-80$ & 70.0 & $0.767 \pm 0.038_{-0.017}^{+0.015}$ & $3.32_{-0.61}^{+0.84}{ }_{-0.30}^{+0.31}$ \\
\hline & & $80-100$ & 90.0 & $0.673 \pm 0.048_{-0.014}^{+0.015}$ & $2.08_{-0.40}^{+0.54}{ }_{-0.13}^{+0.14}$ \\
\hline & & $100-120$ & 110.0 & $0.684 \pm 0.050_{-0.030}^{+0.032}$ & $2.19_{-0.43}^{+0.59}{ }_{-0.27}^{+0.36}$ \\
\hline & & $120-145$ & 130.0 & $0.839 \pm 0.074_{-0.048}^{+0.046}$ & $5.3_{-2.0}^{+5.3+2.5}$ \\
\hline
\end{tabular}

Table 7: The spin-density matrix-element $r_{00}^{04}$ and the ratio of cross sections for longitudinally and transversly polarized photons, $R$, as a function of $W$ for two bins in $Q^{2}$. The first uncertainty is statistical and the second systematic. Due to the transformation given in Eq. (5) the error on the measurement of $R$ is asymmetric. 


\section{ZEUS}

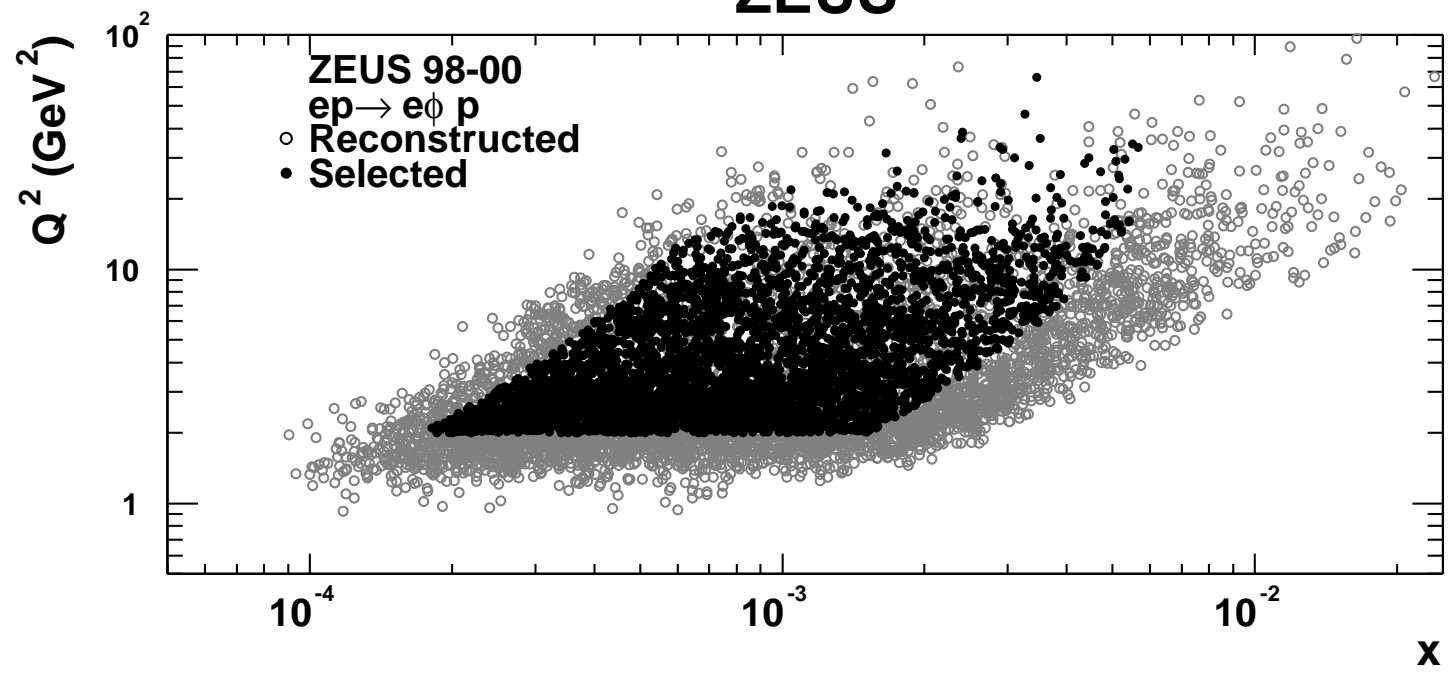

Figure 1: The distribution of the events in the kinematic plane of Bjorken $x$ and $Q^{2}$. The open grey dots represent all reconstructed events while the solid black dots are events in the accepted kinematic range (Section 國). 


\section{ZEUS}

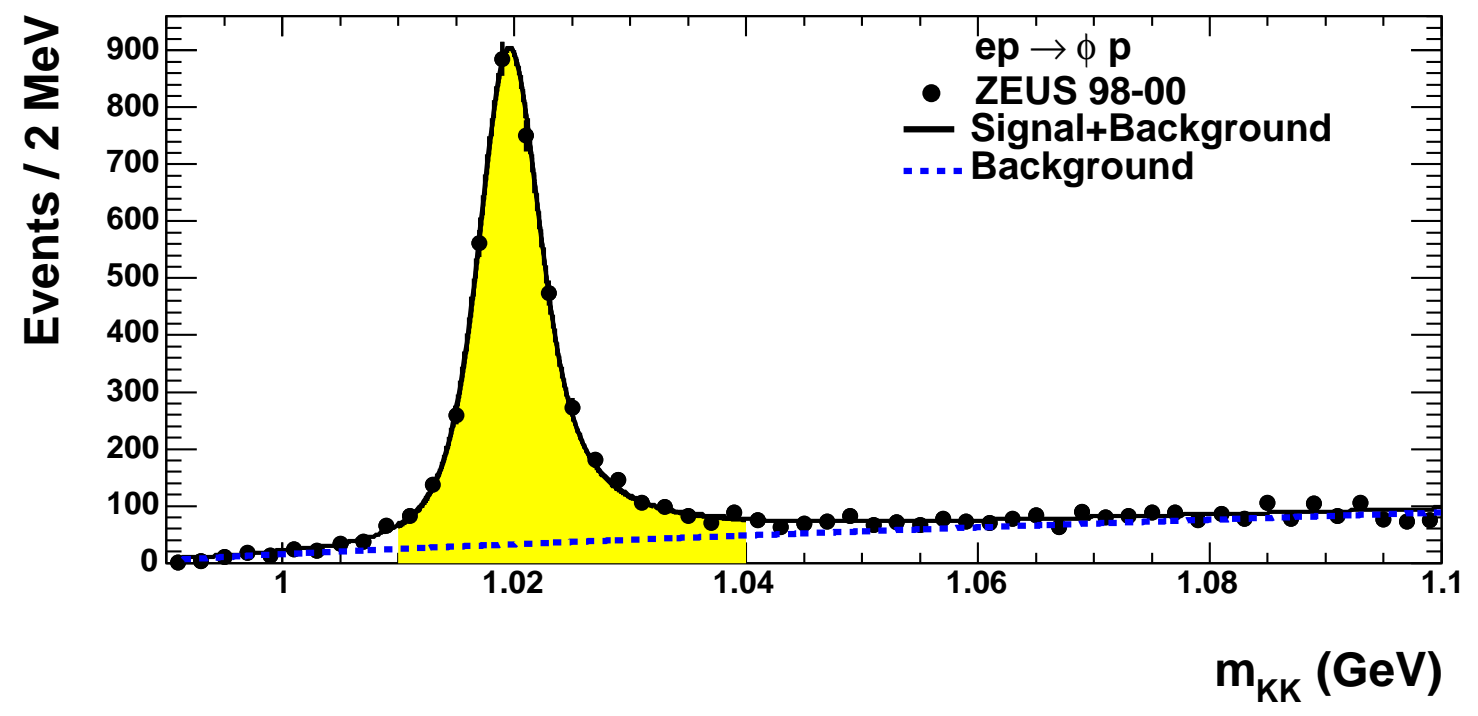

Figure 2: $\quad$ Invariant mass distribution of the $K^{+} K^{-}$candidate pairs. The solid line shows the result of a fit including signal+background, while the dashed line shows the background component only. The shaded region indicates the mass range used for cross-section calculations. The error bars indicate the statistical uncertainties. 
ZEUS

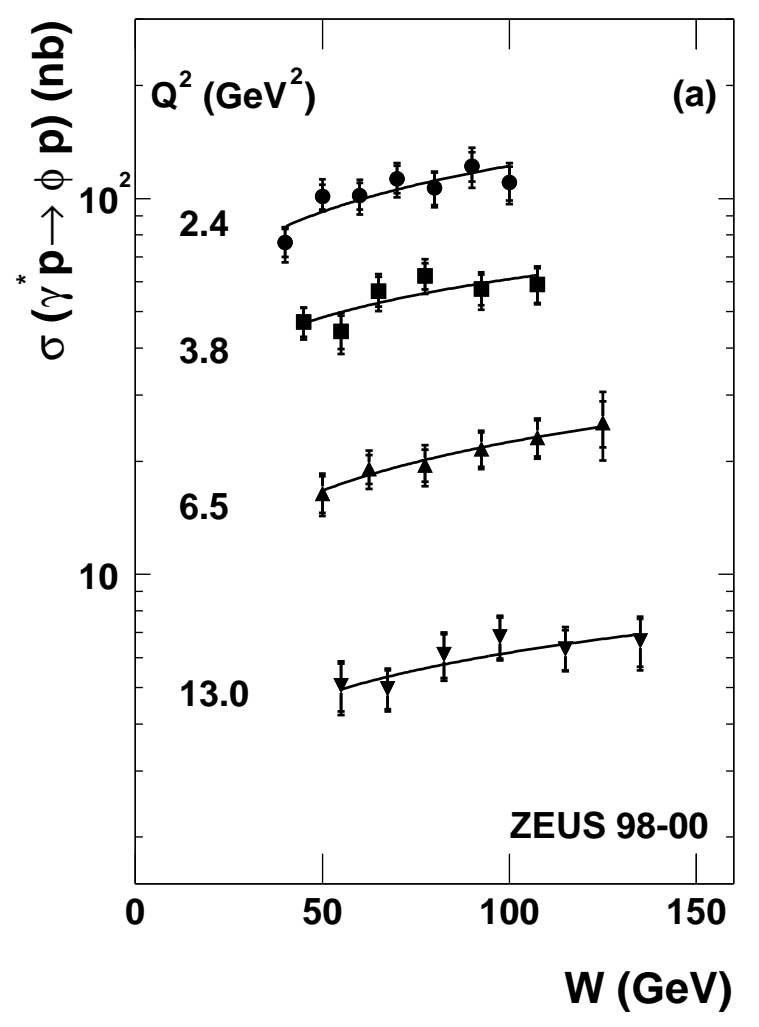

ZEUS

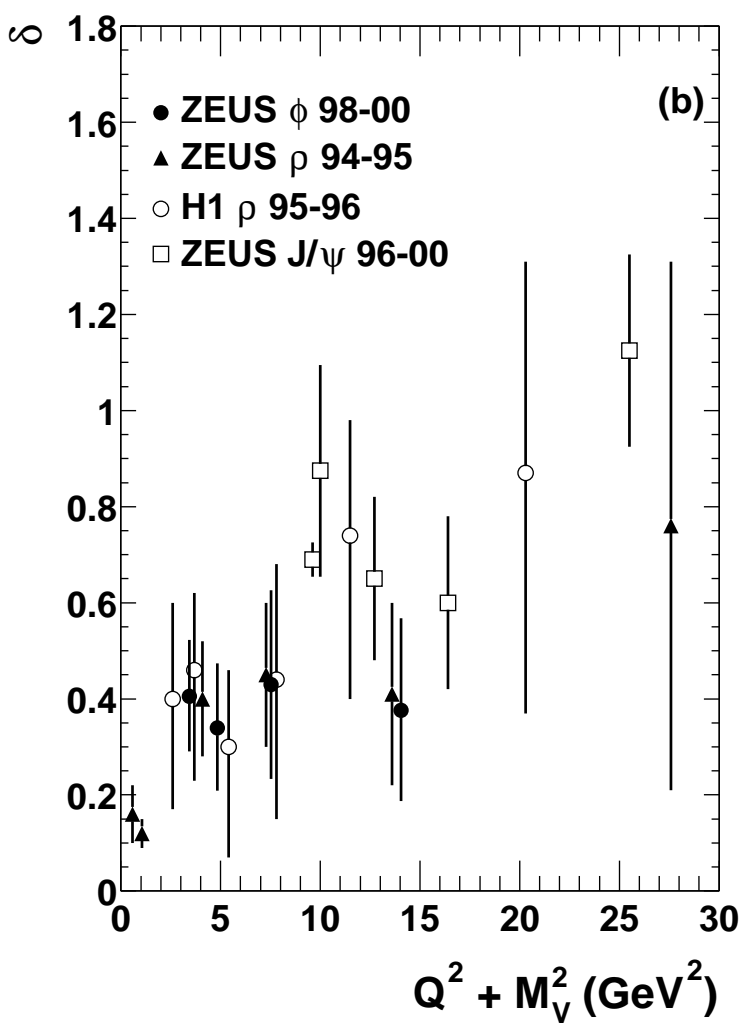

Figure 3: (a) Exclusive $\phi$ cross section as a function of $W$ for four values of $Q^{2}$. The solid lines are the results of a fit to the form $\sigma \propto W^{\delta}$. The overall normalization uncertainty of ${ }_{-5.3}^{+4.1} \%$ is not included in the error bar. (b) The extracted values of $\delta$ compared with results from other vector mesons. The error bars represent the quadratic sum of the statistical and systematic uncertainties. Note that the ZEUS $\rho$ data point at $Q^{2}+M_{V}^{2}$ near $8 \mathrm{GeV}^{2}$ has been shifted down by $0.3 \mathrm{GeV}^{2}$ for clarity of presentation. 


\section{ZEUS}

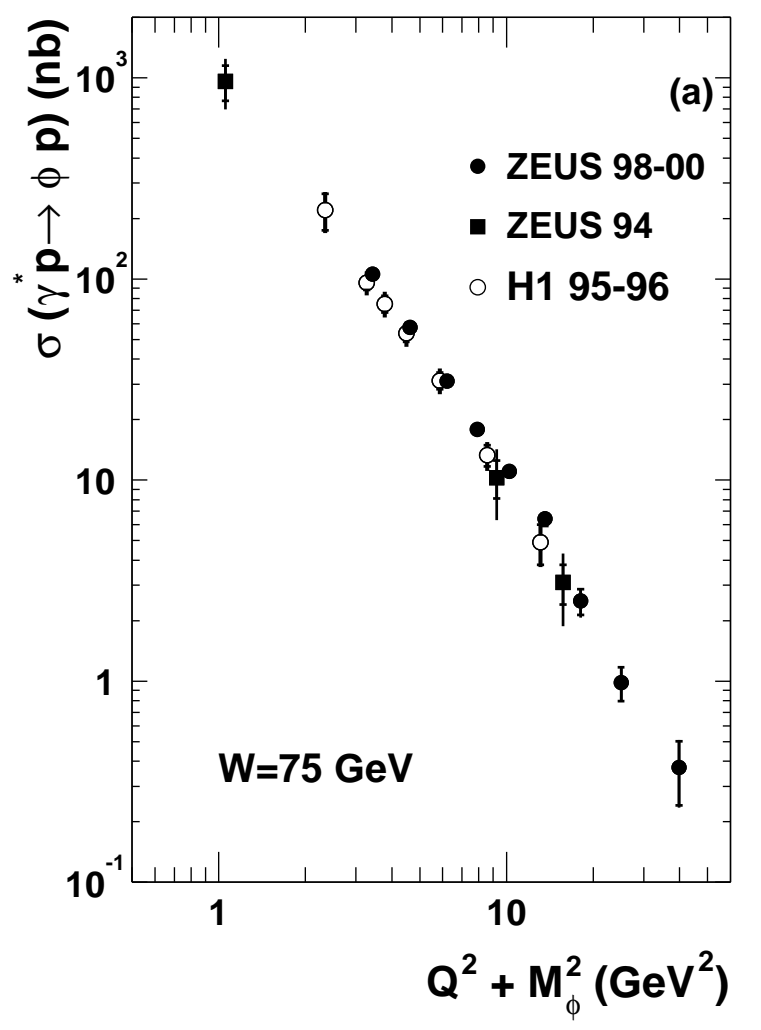

ZEUS

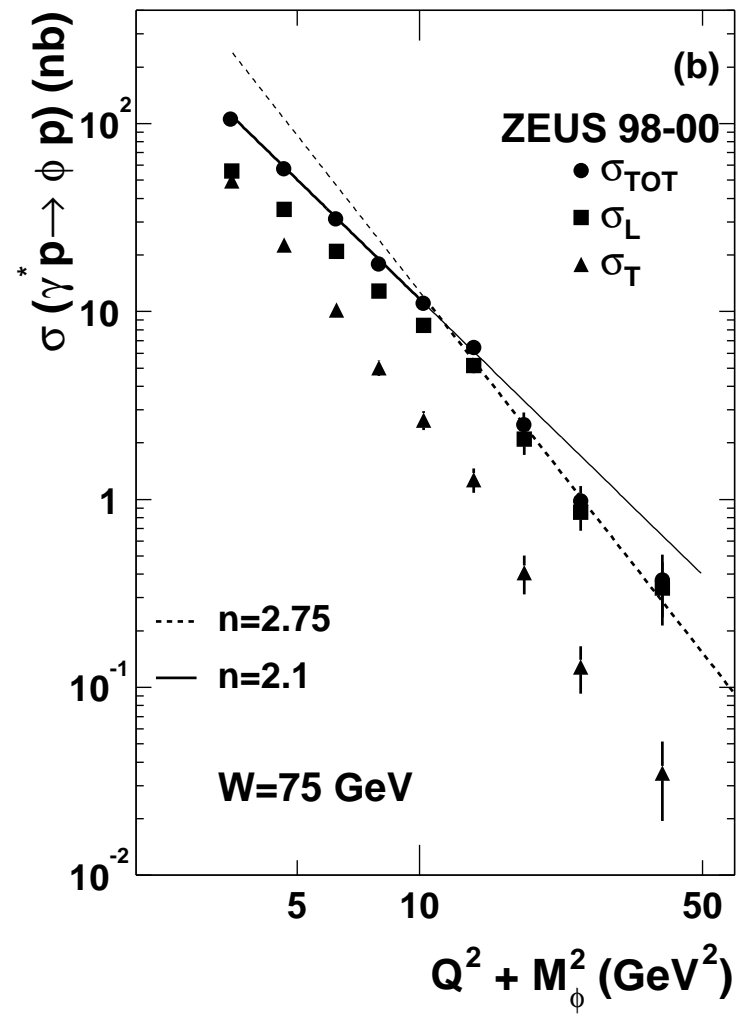

Figure 4: Exclusive $\phi$ cross section as a function of $Q^{2}+M_{\phi}^{2}$ for $W=75 \mathrm{GeV}$. (a) The total cross section compared with previous measurements. (b) The separate longitudinal and transverse contributions to the cross section. The curves represent the results of the fits to the total cross section as described in the text. The error bars represent the quadratic sum of the statistical and systematic uncertainties. The overall normalization uncertainty of ${ }_{-5.3}^{+4.1} \%$ is not shown. 
ZEUS

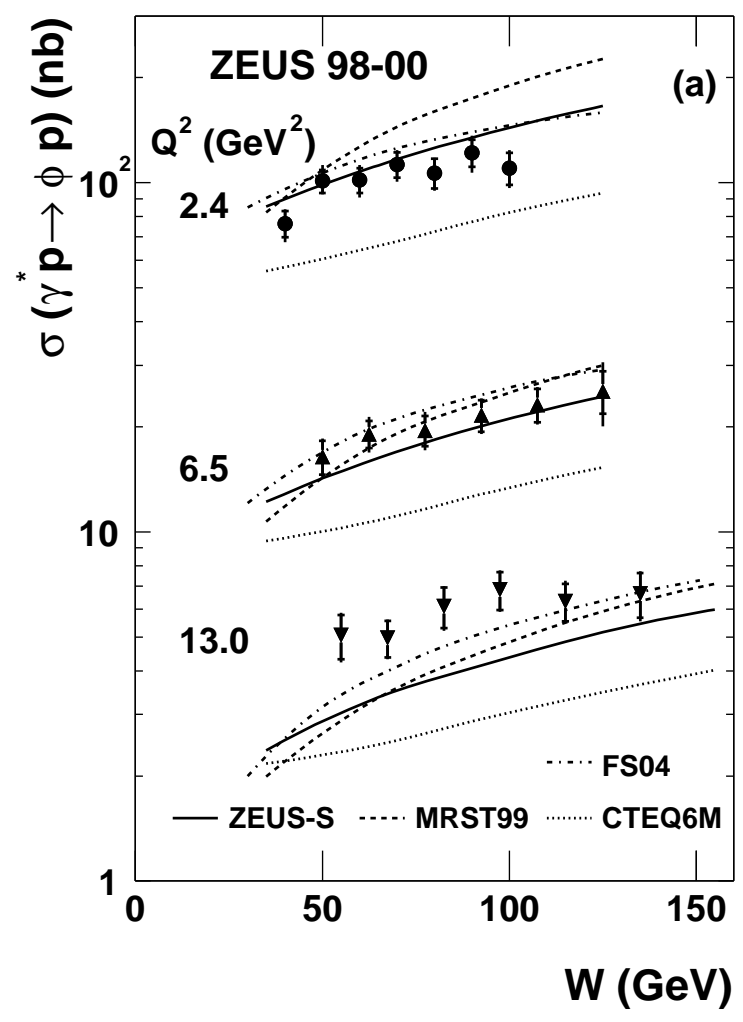

ZEUS

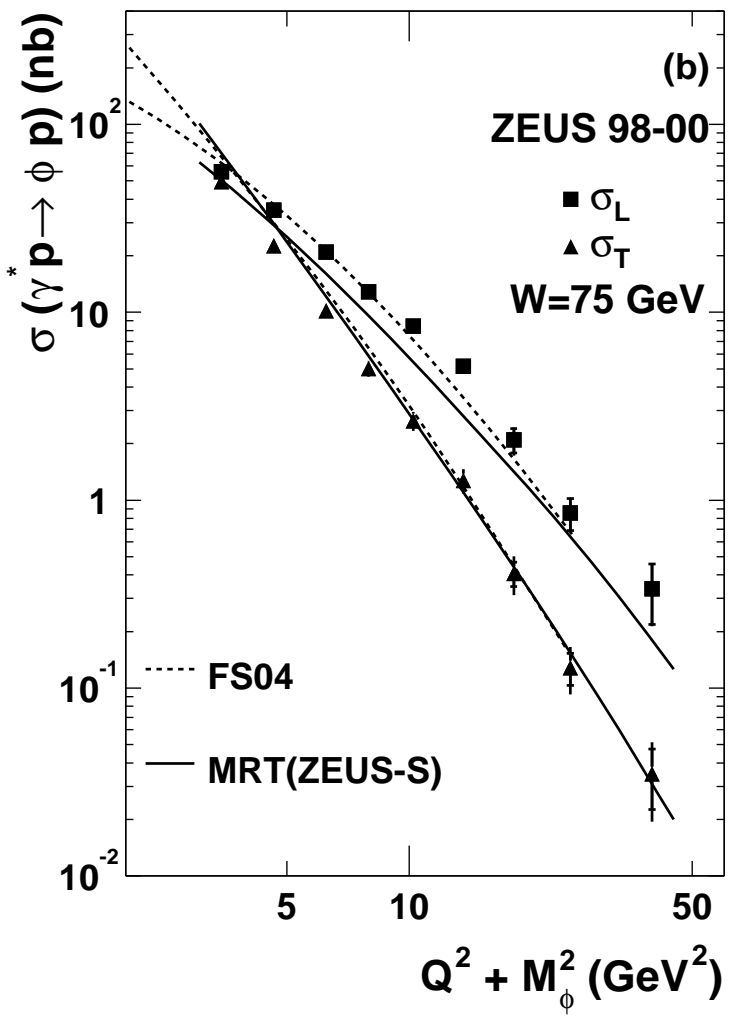

Figure 5: (a) Exclusive $\phi$ cross section as a function of $W$ for three values of $Q^{2}$ compared with predictions from the MRT and FSO4 model. Different gluon densities (ZEUS-S, MRST99, CTEQ6M) were employed in the MRT predictions as indicated in the figure. Note that the data at $Q^{2}=3.8 \mathrm{GeV}^{2}$ are not shown in the figure for clarity. (b) Exclusive $\phi$ cross section as a function of $Q^{2}+M_{\phi}^{2}$ for $W=75 \mathrm{GeV}$. The data are compared to the MRT and FS04 predictions. The ZEUS-S gluon density was used in the MRT model. 
ZEUS

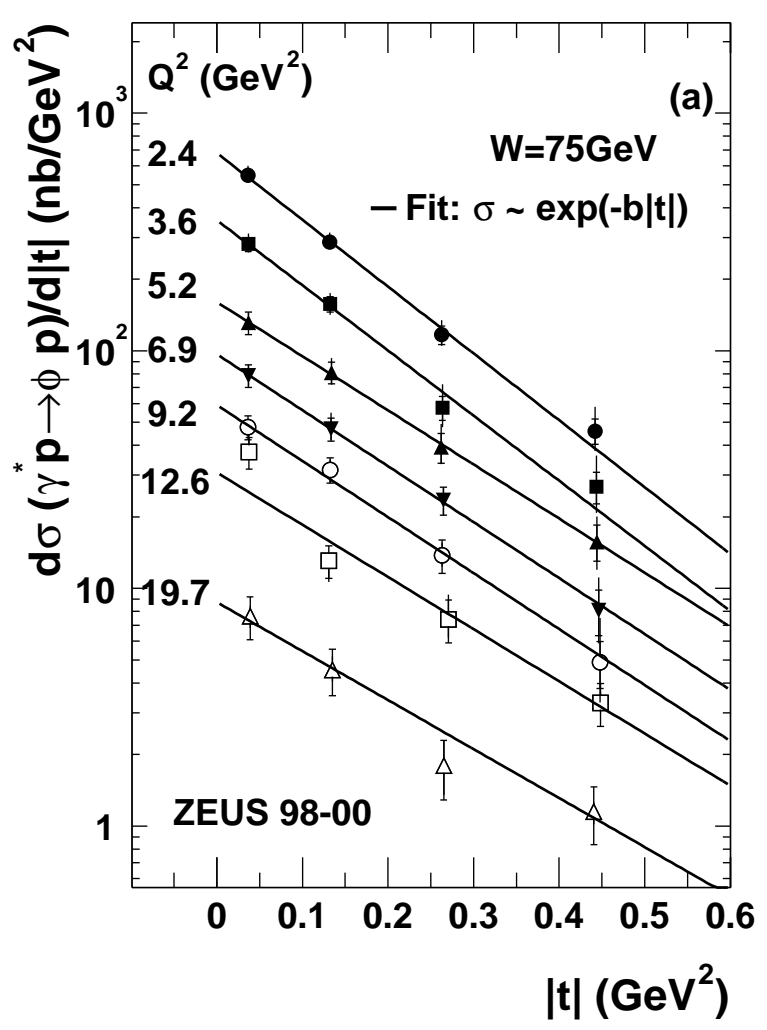

ZEUS

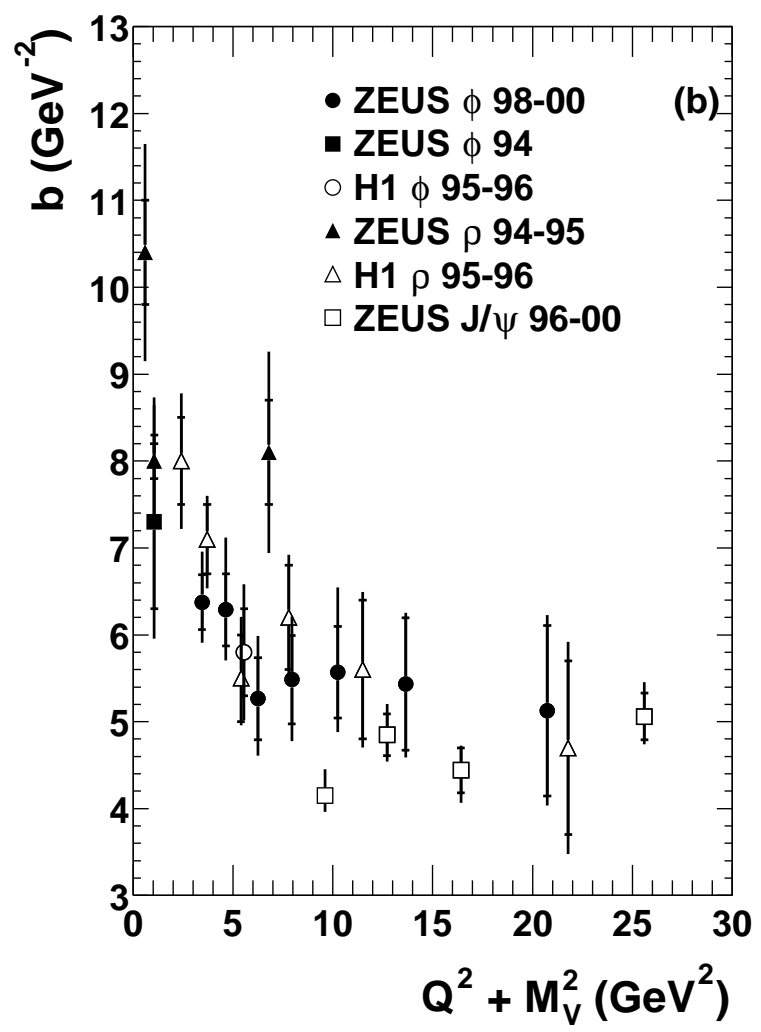

Figure 6: (a) Differential cross-sections $d \sigma / d|t|$ for seven bins of $Q^{2}$, for $W=$ $75 \mathrm{GeV}$ and $|t|<0.6 \mathrm{GeV}^{2}$. The full lines are the results of fits to the form $d \sigma / d t=$ $d \sigma /\left.d t\right|_{t=0} \cdot e^{b t}$. The overall normalization uncertainty of $2.8 \%$ is not included in the error bar. (b) The slope b, as a function of $Q^{2}+M_{V}^{2}$, compared to other ZEUS and $H 1$ results. The inner error bars represent the statistical uncertainty; the outer bars are the statistical and systematic uncertainties added in quadrature. 
ZEUS

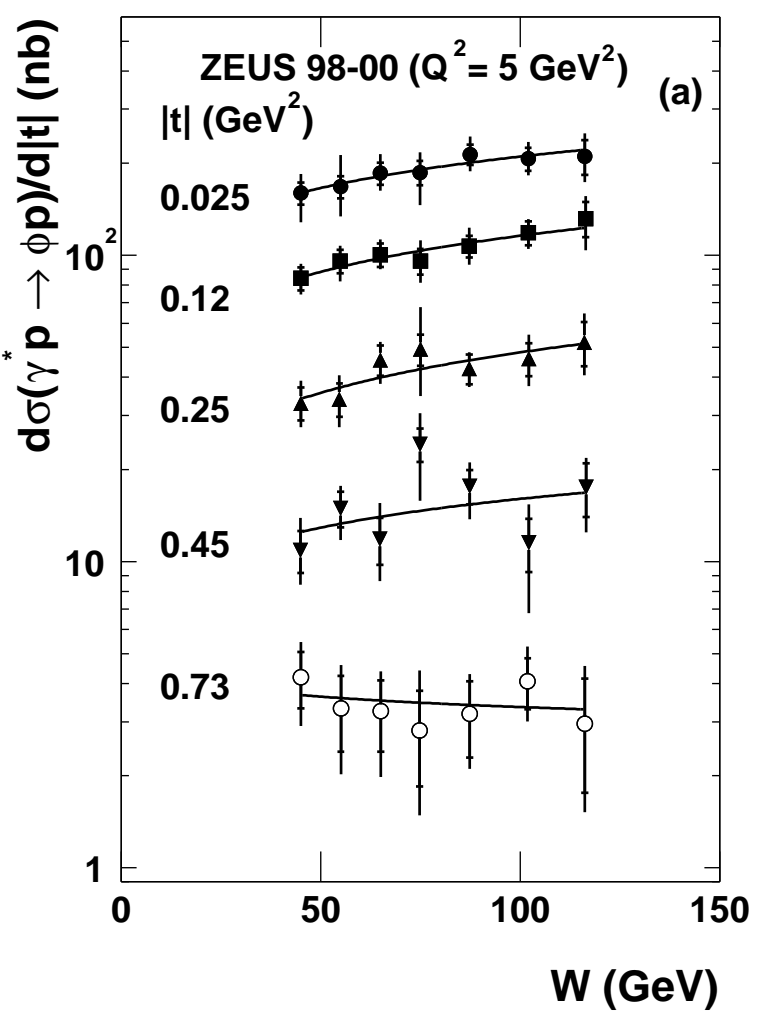

ZEUS

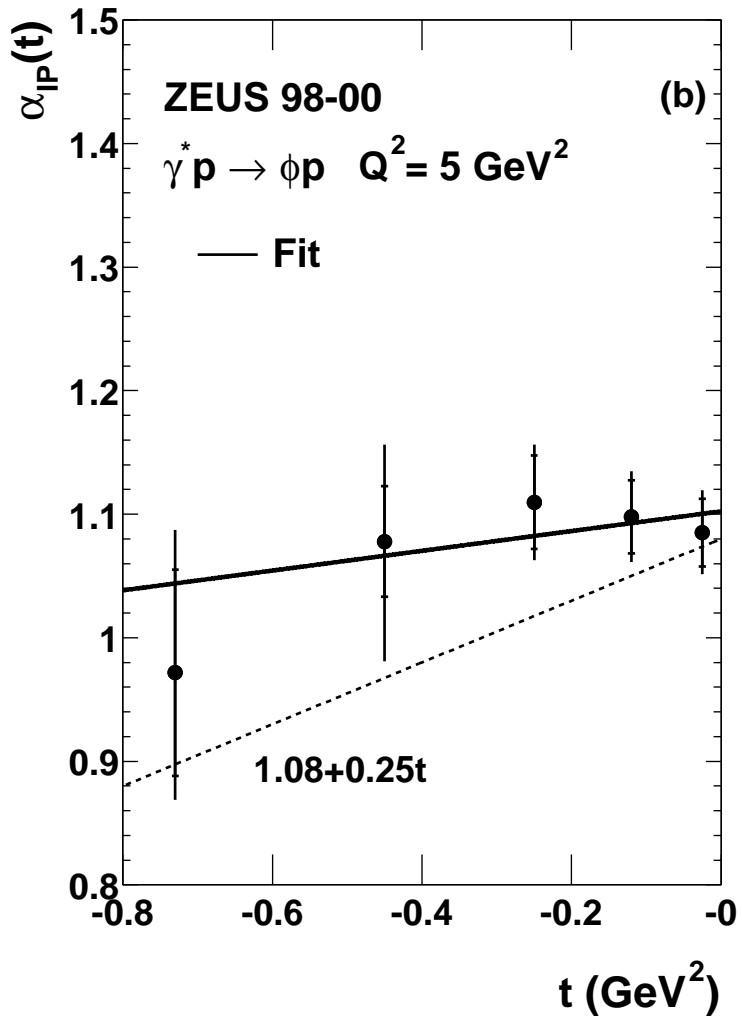

Figure 7: (a) Differential cross-sections $d \sigma / d|t|$ as a function of $W$ for different values of $t$ at $Q^{2}=5 \mathrm{GeV}^{2}$. The full lines are fits to the form $W^{\delta}$. The mean contribution from proton dissociation has been subtracted. The overall normalization uncertainty of ${ }_{-5.3}^{+4.1} \%$ is not shown. (b) The extracted values of $\alpha_{\mathbb{P}}$. The full line is a linear fit to $\alpha_{\mathbb{P}}$ with the form given in Eq.(3). The dotted line is the soft Pomeron trajectory [22]. The inner error bars represent the statistical uncertainty; the outer bars are the statistical and systematic uncertainties added in quadrature. 


\section{ZEUS}
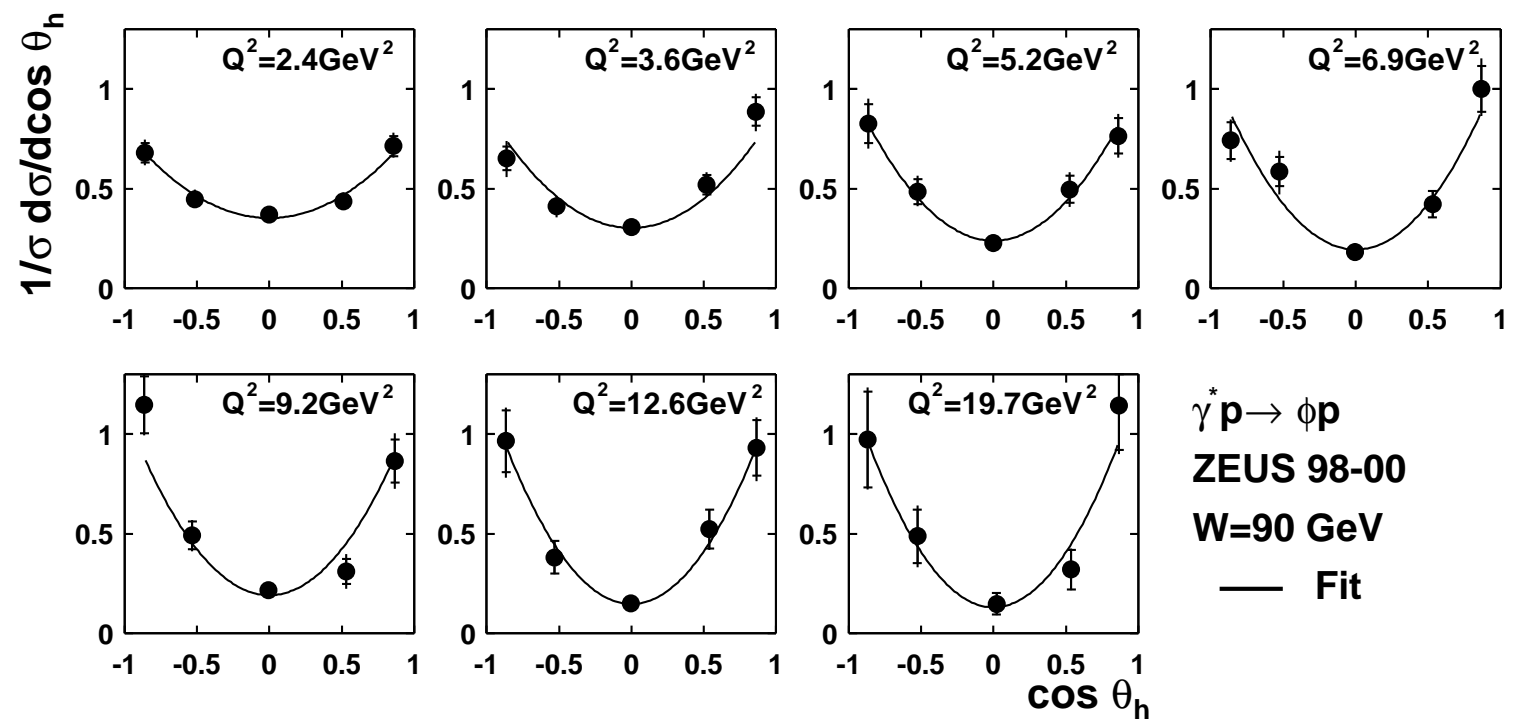

$\gamma^{*} \mathbf{p} \rightarrow \phi \mathbf{p}$

ZEUS 98-00

$\mathrm{W}=90 \mathrm{GeV}$

- Fit

Figure 8: Normalized distributions of $\cos \theta_{h}$ in seven $Q^{2}$ bins; the curves are the fits to Eq. (4). The inner error bars represent the statistical uncertainty; the outer bars are the statistical and systematic uncertainties added in quadrature.

\section{ZEUS}

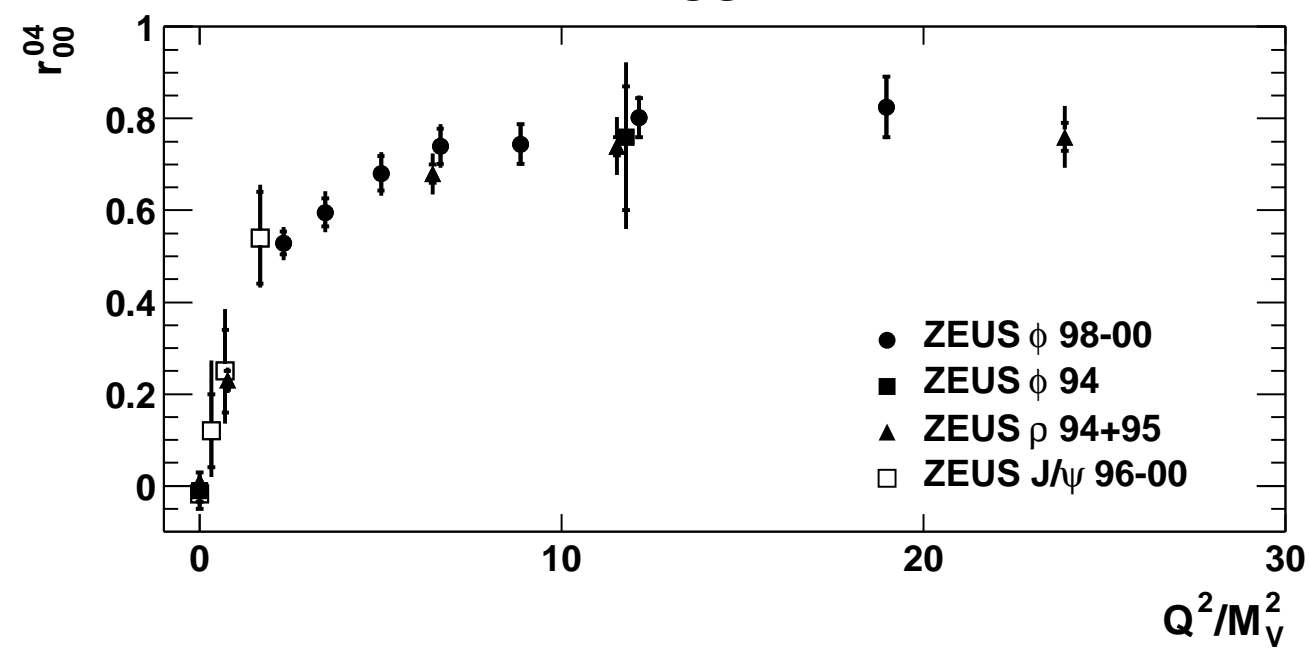

Figure 9: The values of $r_{00}^{04}$ extracted from the fits shown in Fig. 8 are plotted as a function of $Q^{2} / M_{V}^{2}$ and compared with values for other vector mesons. 
ZEUS

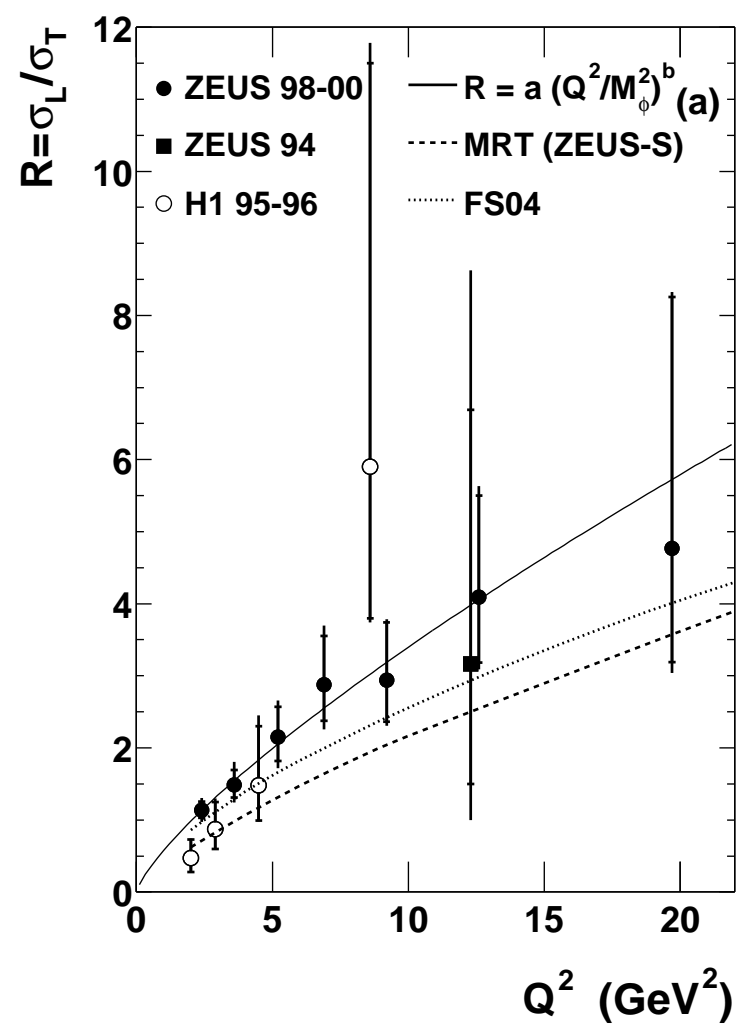

ZEUS

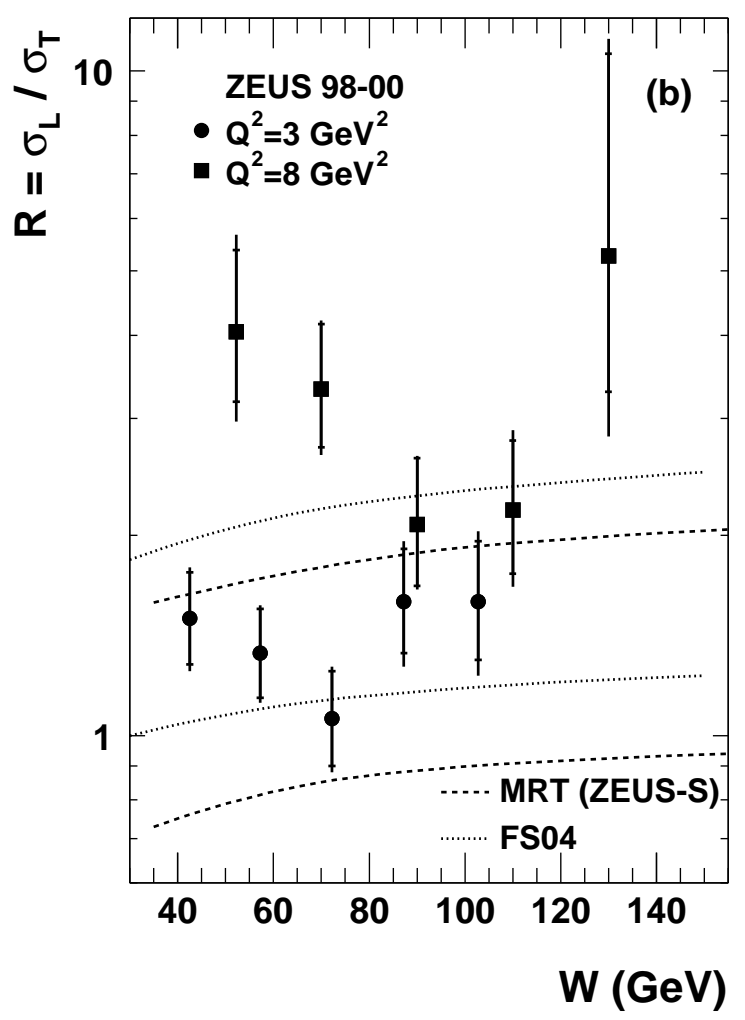

Figure 10: (a) Ratio $R=\sigma_{L} / \sigma_{T}$ as a function of $Q^{2}$ for exclusive $\phi$ production; the full line is the result of the two-parameter fit as shown in the plot. (b) Ratio $R=\sigma_{L} / \sigma_{T}$ as a function of $W$ extracted in this analysis for two different $Q^{2}$ values. The dashed curve in both plots is from the MRT model with the ZEUS-S gluon density, while the dotted curve is from the FSO4 model. The inner error bars show the statistical uncertainty, the outer the statistical and systematic uncertainties added in quadrature. 